NBER WORKING PAPER SERIES

\title{
EQUILIBRIUM TRADE IN AUTOMOBILE MARKETS
}

\author{
Kenneth Gillingham \\ Fedor Iskhakov \\ Anders Munk-Nielsen \\ John P. Rust \\ Bertel Schjerning \\ Working Paper 25840 \\ http://www.nber.org/papers/w25840
NATIONAL BUREAU OF ECONOMIC RESEARCH
1050 Massachusetts Avenue
Cambridge, MA 02138
May 2019

We dedicate this paper to James A. Berkovec whose contributions to the development of microfounded equilibrium models of the auto market was far ahead of his time and so inspirational to our own work on this subject. His untimely death at age 52 in 2009 remains a huge loss to the economics profession. The authors would like to acknowledge funding from the IRUC research project, financed by the Danish Council for Independent Research. Rust acknowledges financial support from the Gallagher Family Chair in Economics at Georgetown University. The views expressed herein are those of the authors and do not necessarily reflect the views of the National Bureau of Economic Research.

NBER working papers are circulated for discussion and comment purposes. They have not been peer-reviewed or been subject to the review by the NBER Board of Directors that accompanies official NBER publications.

(C) 2019 by Kenneth Gillingham, Fedor Iskhakov, Anders Munk-Nielsen, John P. Rust, and Bertel Schjerning. All rights reserved. Short sections of text, not to exceed two paragraphs, may be quoted without explicit permission provided that full credit, including (C) notice, is given to the source. 
Equilibrium Trade in Automobile Markets

Kenneth Gillingham, Fedor Iskhakov, Anders Munk-Nielsen, John P. Rust, and Bertel Schjerning NBER Working Paper No. 25840

May 2019

JEL No. L9,L98,Q4,Q5,R4

\begin{abstract}
We introduce a computationally tractable dynamic equilibrium model of the automobile market where new and used cars of multiple types (e.g. makes/models) are traded by heterogeneous consumers. Prices and quantities are determined endogenously to equate supply and demand for all car types and vintages, along with the ages at which cars are scrapped. The model allows for transactions costs, taxes, flexible specifications of car characteristics, consumer preferences, and heterogeneity. We apply the model to two examples: a revenue-neutral replacement of the new vehicle registration tax with a higher fuel tax and a hypothetical "merger to monopoly" in an oligopolistic new car market. We show substantial gains in consumer welfare from the tax policy change, as well as important effects on government revenues, automobile prices, driving, fuel consumption and $\mathrm{CO}_{2}$ emissions, while the merger leads to substantial welfare losses.
\end{abstract}

Kenneth Gillingham

School of Forestry and Environmental Studies

Yale University

195 Prospect Street

New Haven, CT 06511

and NBER

kenneth.gillingham@yale.edu

Fedor Iskhakov

Australian National University

Research School of Economics

H.W. Arndt Building

25a Kingsley P1.

Acton ACT 2601

Australia

fedor.iskhakov@anu.edu.au

Anders Munk-Nielsen

University of Copenhagen

Department of Economics

Oster Farimagsgade 5, 26.3.01

DK-1353 Copenhagen K, Denmark

superpronker@gmail.com
John P. Rust

Georgetown University

583 Intercultural Center

Washington, DC 20057

jr1393@georgetown.edu

Bertel Schjerning Department of Economics University of Copenhagen Øster Farimagsgade 5, building 26

DK-1353 Copenhagen K. bertel.schjerning@econ.ku.dk 


\section{Introduction}

We present a computationally tractable dynamic equilibrium model of the automobile market where new and used vehicles of multiple types (e.g. makes and models) are traded by heterogeneous consumers. The prices and quantities of used cars are determined endogenously to equate supply and demand for all car types and traded vintages, and the ages at which cars are scrapped are also determined endogenously as part of the equilibrium. The model allows for transactions costs, taxes, and flexible specifications of car characteristics, consumer preferences, and heterogeneity, allowing for a rich analysis of a suite of research questions that involve both primary and secondary markets for vehicles.

Our approach is based on a dynamic discrete choice model of ownership and trading of automobiles where a key aspect of our specification of consumer heterogeneity is the inclusion of additive idiosyncratic extreme value components to the utilities from the different possible choices individuals face. Such preference shocks have a clear economic motivation, since they can represent the idiosyncratic random components of the cost of maintaining an existing car, as well as idiosyncratic aspects of the costs of search, prices of cars, and transactions costs. These shocks constitute a fundamental source of gains from trade that rationalize the existence of secondary markets. By varying the scale of these additive extreme value preference shocks, we show how reductions in consumer heterogeneity reduce gains from trade and ultimately kill off secondary markets when there are sufficiently large trade frictions.

An advantage of our framework is that the extreme value specification results in convenient functional forms for the conditional choice probabilities for the decisions to keep or trade different types and ages of vehicles. Thus, we have the flexibility to match rich patterns of trading, including consumers who choose not to own cars (i.e. who choose the "outside good") and "brand loyalty" and "brand switching" behaviors. We show that the choice probabilities are smooth functions of car prices, and thus excess demands are also smooth functions of prices. This allows us to use a robust and efficient algorithm, Newton's Method, to solve for consumers' dynamic trading strategies as well as equilibrium prices.

We formulate our model and define equilibrium in an infinite horizon stationary environment. We use the machinery of Markov processes to describe trading behavior and characterize the vehicle holdings of different types of consumers as invariant distributions to certain Markov chains. These Markov chains reflect the trading of vehicles, their aging and the im- 
pact of stochastic accidents that result in premature scrappage of some vehicles. Our stationary equilibrium concept results in a very compact and elegant description of equilibrium, but it can also be extended to non-stationary environment (e.g. with macroeconomic shocks) or an "overlapping generations" framework with finite individual lifespans. Since it is substantially more complicated to define and calculate equilibrium in these various extensions, we introduce our approach in the stationary, infinite horizon setting where we can define and characterize the equilibrium more elegantly and simply.

The primary contribution of this paper is methodological: we develop a new framework for computing equilibrium in the primary and secondary automobile markets. To demonstrate the usefulness of our model, we examine two stylized examples. The first is motivated by the new vehicle registration taxes in Denmark, which are some of the highest in the world: for the majority of new vehicles sold today, it is as high as $150 \% .^{1}$ The registration tax discourages new vehicle purchases, and leads to a fleet of older vehicles on the road. Thus, there has been great policy interest in alternatives. Using data from Denmark, we calibrate the model to the Danish economy and solve for the counterfactual equilibrium following a revenue-neutral reform that reduces the new vehicle registration tax and increases the fuel tax. We examine the effects on government revenues, automobile prices over the age distribution of vehicles, driving, $\mathrm{CO}_{2}$ emissions and consumer welfare, and find substantial gains in consumer welfare from the tax change, but also a substantial increase in fuel consumption and $\mathrm{CO}_{2}$ emissions due to extensive margin responses in car ownership and vehicle size.

For our second stylized example, we endogenize new vehicle prices, allowing us to examine a hypothetical "merger to monopoly" where two firms in a Bertrand-Nash equilibrium merge. We show existence of the unique duopoly Bertrand-Nash equilibrium and then examine how prices, profits, and consumer surplus change when the two firms merge. After the merger, the monopolist dramatically increases prices, which decreases sales and increases firm profits but at the expense of consumer and total surplus, which are nearly halved.

These two examples highlight the power of our approach. This framework has potential to be useful for understanding the full equilibrium effects of many policies, including vehicle registration fees, gasoline taxes, per-kilometer usage charges, and low-emissions vehicle mandates. Moreover, the flexibility and tractability of the model makes it promising for use in

\footnotetext{
${ }^{1}$ Denmark also imposes fuel taxes and vehicle ownership taxes, but the registration tax is by far the largest revenue-raiser, bringing in between 30 and 50 billion kroner (DKK) annually.
} 
structural estimation, potentially allowing for a wide range of policy analyses. ${ }^{2}$

A starting point of any discussion of the literature on equilibrium models of automobile markets is the well-known BLP model (Berry, Levinsohn and Pakes, 1995). This influential work focuses on the primary market for new vehicles, and ignores the presence of the secondary market and the substitution possibilities it offers consumers. Rust (1985c) and Esteban and Shum (2007) were the first to tackle the challenging problem of solving for a full equilibrium in both the primary and secondary markets for automobiles. Rust studied the simultaneous determination of price and durability by a monopolist new vehicle producer, while Esteban and Shum studied oligopolistic pricing of competing new vehicle producers. To make progress, both of these studies assumed stationarity and zero transaction costs, which implies that consumer trade each period for their most preferred vehicle in the entire market. ${ }^{3}$

We introduce a dynamic equilibrium model of automobile trading in the primary and secondary markets for automobiles with heterogeneous consumers and transactions costs. In equilibrium, consumers will "buy and hold" and only an endogenously determined subset of consumers will buy new vehicles each period. An important feature of our model is that we can characterize the subset of consumers who are most likely to trade, as well as the characteristics of the subsets of the population most likely to buy and hold different types of vehicles.

We build on a substantial literature focused on modeling equilibrium in secondary markets for automobiles, taking the price of new vehicles as given. The earliest work that we are aware of in this literature is a series of papers by Manski (1980), Manski and Sherman (1980), and Manski (1983). These papers introduced theoretical models of equilibrium in secondary markets for cars that could be numerically solved for prices and quantities and used for policy forecasting of a wide range of policies of interest.

Another important early contribution was Berkovec (1985), who microeconometrically estimated and numerically solved a large-scale equilibrium model of the new and used vehicle markets using a nested logit model. He defined "expected excess demand" by summing estimated discrete choice probabilities for cars of each age and class, net of scrappage. ${ }^{4}$ Berkovec

\footnotetext{
${ }^{2}$ In related on-going work, Gillingham, Iskhakov, Munk-Nielsen, Rust and Schjerning (2019) are structurally estimating an overlapping generations model using a similar framework.

${ }^{3}$ Esteban and Shum (2007) also assume quality ladder preferences, which further simplifies the choice problem.

${ }^{4}$ Berkovec used a probabilistic model of vehicle scrappage from Manski and Goldin (1983), where the probability a vehicle is scrapped is a decreasing function of the difference between the second-hand price of the vehicle (net of any repair costs) and an exogenously specified scrap value for the vehicle. This implies that, except for random accidents, there is very little chance that new vehicles are scrapped, but the probability a used vehicle is scrapped increases monotonically with the age of the vehicle.
} 
computed equilibrium prices using Newton's method to find a zero to a system of 131 nonlinear equations representing the excess demand for the vehicles in his model. ${ }^{5}$

The contributions of Manski, Sherman, and Berkovec were extremely advanced given the computing power at the time, and in many respects represent the closest point of departure for our own work. However, their work was based on short run, static equilibrium holding models of the market. Implicit in the static discrete choice formulation is the assumption that consumers only keep their vehicle for a single period, so that at the end of each period consumers trade their current vehicle for their most preferred vehicle. Rust (1985b) formulated the first dynamic model of automobile trading. He assumed the state of a vehicle is captured by its odometer reading $x_{t}$, which evolves according to an exogenous Markov process representing variable usage of cars with transition probability $\Phi\left(x_{t+1} \mid x_{t}\right)$ that reflects stochastic usage and deterioration of vehicles. ${ }^{6}$

When there are no transaction costs and the economy is in a stationary equilibrium (i.e. no macroeconomic shocks or other time-varying factors altering the market), the optimal trading strategy involves trading every period for the most preferred age/condition of vehicle $x^{*}(\tau)$, where $\tau$ indexes potentially heterogeneous preferences over "newness" of vehicles. However, the assumption of zero transactions costs is unrealistic, and so is the excessive trading behavior it implies. When there are transactions costs (which are distinct from trading costs, i.e. the difference between the price of a car $x$ a consumer wishes to buy, $P(x)$, less the price $P\left(x^{\prime}\right)$ of the car $x^{\prime}$ that the consumer wishes to sell), the optimal trading strategy involves less frequent trading and consumers will generally keep cars for multiple periods. The optimal strategy then takes the form of an " $(S, s)$ rule" reminiscent of optimal inventory theory: trade is characterized by two thresholds $\left(\underline{x}^{*}(\tau), \bar{x}^{*}(\tau)\right)$, where $\underline{x}^{*}(\tau)<\bar{x}^{*}(\tau)$ and $\underline{x}^{*}(\tau)$ is the state of the optimal replacement vehicle whenever the consumer trades in for a new one. $\bar{x}^{*}(\tau)$ is the replacement threshold or odometer threshold where it is optimal to trade the current car in condition $x$ for a replacement car in condition $\underline{x}^{*}(\tau)$. Of course, when transactions costs are zero, then $\bar{x}^{*}(\tau)=$ $\underline{x}^{*}(\tau)=x^{*}(\tau)$ and it is optimal to trade for the optimal car $x^{*}(\tau)$ every period. However, in a homogeneous agent economy, transaction costs will completely kill off trading in the secondary market, so that all consumers follow an extreme version of a "buy and hold" strategy: they buy a

\footnotetext{
${ }^{5}$ Berkovec showed that the Jacobian matrix had special structure he called "identity outer product" that enabled him to invert the Jacobian via inverting a smaller $48 \times 48$ matrix and doing some additional matrix vector multiplications.

${ }^{6}$ Since $x_{t}$ fully captures the state of a car and is observable by both parties in a transaction, Rust's analysis avoided "lemons problem" information asymmetries of the type analyzed in the seminal work of Akerlof (1970) that can potentially kill off the secondary markets for cars.
} 
new vehicle whenever they trade (i.e. $\underline{x}^{*}(\tau)=0$ ) and scrap their existing one when its odometer reaches the replacement threshold $\bar{x}^{*}(\tau)$. This too is unrealistic.

Moving to a heterogeneous agent economy can allow for more flexible strategies, but establishing the existence of a stationary equilibrium in such an economy in the presence of transactions costs is challenging. Consider a consumer of type $\tau$ who desires to buy a vehicle with $\underline{x}^{*}(\tau)>0$. When there are transactions costs there is no guarantee that some other consumer $\tau^{\prime}$ is willing to sell their vehicle at $\underline{x}^{*}(\tau)$. Using advanced methods from functional analysis (e.g. the Fan-Glicksburg fixed point theorem), Konishi and Sandfort (2002) established the existence of a stationary equilibrium in the presence of transactions costs under certain conditions. Their proof shows that it is possible for the equilibrium price function $P(x)$ to adjust to prevent such coordination failures. However to our knowledge, there has been no work actually calculating equilibria with transactions costs in this infinite-dimensional setting.

Stolyarov (2002) advanced the literature by assuming that the state of a vehicle can be summarized by its age $a$, which can take only a finite number of values, $a=0,1,2, \ldots, \bar{a}$, where $\bar{a}$ scrappage age. Stolyarov introduced a continuous uni-dimensional parameterization of consumer heterogeneity with quasi-linear preferences, and computed equilibria in the presence of stochastic transactions costs using a fixed point formulation of the problem. Gavazza, Lizzeri and Roketskiy (2014) extended Stolyarov's approach by allowing households to own up to two vehicles using a two-dimensional specification of consumer heterogeneity. They find that transaction costs have a large effect on equilibrium trade.

Our model can be thought of as combining Stolyarov (2002) with the earlier work by Manski and Berkovec by using a multi-dimensional extreme value specification of consumer heterogeneity. We use a hierarchical specification of heterogeneity that includes both time-varying idiosyncratic preference shocks (i.e. the extreme value error terms in the model) as well as flexible specifications for time-invariant heterogeneity or types $\tau$. The extreme value distribution allows for continuous formulas for choice probabilities even in the case where there is no other time-invariant heterogeneity, and this continuity permits us to demonstrate the existence of equilibrium via the Brouwer fixed point theorem. More importantly, we show that the excess demand function for used cars in our model, $E D(P)$, is a continuously differentiable function of $P$ that enables us to rapidly and accurately calculate equilibrium prices by solving the system of nonlinear equations $E D(P)=0$ by Newton's method. This makes our approach very attractive for use in empirical work and policy modeling. 
There is a close connection between models of automobile trading that incorporate transactions costs and models that emphasize information asymmetries, such as Akerlof (1970). There are "lemons laws" in many countries that require sellers to compensate buyers for defects or problems in a car that were not disclosed and negotiated on at the time of sale. Dealers typically perform inspections and repair cars before selling, and often provide a limited term warranty, all of which mitigate the informational asymmetries and result in transactions costs that are often borne by the dealer. As a result, it is not clear that informational asymmetries seriously inhibit trade in used vehicles, but they would be expected to show up in transaction costs. Hendel and Lizzeri (1999) study equilibria in auto markets with and without asymmetric information and find that adverse selection does not necessarily kill off the secondary market. They find it difficult to empirically distinguish between predictions of models with asymmetric information and those with transaction costs, and argue that, for Fords and Hondas at least, the evidence does not support adverse selection as the primary reason for steeper price declines of Fords as the vehicles age. In light of this, we move forward in using transaction costs to capture trade frictions in auto markets.

In the next several sections we build up the computational framework for modeling the automobile market. In Section 2 we set up the basic model with one car type and idiosyncratic consumer heterogeneity. In Section 3 we consider persistent consumer heterogeneity to study equilibrium sorting of cars between different consumer types. In Section 4 we extend the model to consider a market with multiple car makes/models. Section 5 calibrates this model to the Danish setting for a stylized exploration into the change in Danish tax policy. Finally, in Section 6 we consider Bertrand competition between multiple-product car producers, thus endogenizing the new car prices and allowing us to analyze the effects of a hypothetical "merger to monopoly" counterfactual. Section 7 concludes.

\section{Equilibrium with Idiosyncratic Consumer Heterogeneity}

In this section we introduce a dynamic model of equilibrium trade at the automobile market. We consider a stationary equilibrium in an infinite horizon economy where aging cars are traded in both new and used car markets, and where consumers make replacement, trading and ownership decisions to maximize expected discounted utility with a common discount factor $\beta \in(0,1)$. For simplicity, we initially consider a single car type $j=1$ so that cars differ only by their 
age $a \in\{1, \ldots, \bar{a}\}$ and a unit mass of idiosyncratically heterogeneous consumers who differ by their preferences over these cars as well as the outside option of not owning a car. We adopt an extreme value specification of time-varying consumer heterogeneity that results in a logit specification for choice probabilities similar to Berkovec (1985) that allows for both tractable and realistic modeling of equilibrium trade in an economy with both transaction costs and the outside option not owning a car.

\subsection{The model and key assumptions}

We focus on the stationary environment, and do not allow any "macro shocks" such as timevarying fuel price or price of new cars. We use the concept of stationary equilibrium from Rust (1985b) and adapt it to our discrete product market setting. Under this concept there is a time invariant price function $P(a)$ satisfying $P(0)=\bar{P}, P(a)=\underline{P}$ for $a \geq \bar{a}$, and for each $a \in\{1, \ldots, \bar{a}-1\}, P(a)$ equates the supply of cars of age $a$ sold by existing consumers in the secondary market to the demand for cars of age $a$ by other consumers in the economy. We initially treat the model as a "small open economy" where new car prices are determined in the world market with an infinitely elastic supply of new cars at price $\bar{P}$. We also assume there is an infinitely elastic demand for cars for their scrap value $\underline{P}$ such that $P(a) \geq \underline{P}$.

Similar to Rust (1985b), the scrap age $\bar{a}$ is endogenously determined in equilibrium as the smallest age where $P(a)$ equals $\underline{P}$. In Appendix A we show that the key results from the continuum of goods setting of Rust (1985b) extend to our finite good setting, including the result that in equilibrium the scrappage age $\bar{a}$ coincides with the optimal replacement threshold to a social planner's problem, and is therefore welfare maximizing. We show how to compute $\bar{a}$ together with equilibrium prices $P(a)$ through an iterative search algorithm that finds the maximum value of $\bar{a}$ that can be supported in equilibrium. ${ }^{7}$

We assume that the marginal utility of an additional car is sufficiently small such that no consumer would want to own more than a single car. ${ }^{8}$ We further assume that make/model $j \in\{1, \ldots, J\}$ reflects all persistent differences between the car types, and that car age $a \in$ $\{1, \ldots, A\}$ reflects all of its time-varying characteristics. Until Section 4 we set $J=1$ (and omit

\footnotetext{
${ }^{7} \mathrm{We}$ can interpret the equilibrium outcome with highest supported $\bar{a}$ as the outcome of a decentralized trading process where the used price $P(a)$ is bid up whenever there is an excess demand for a car of age $a$, and conversely $P(a)$ falls whenever there is an excess supply for cars of age $a$.

${ }^{8}$ This is a reasonable assumption for a country like Denmark, where most households only own a single car. It also greatly simplifies notation and the presentation of our model. The assumption could be relaxed to extend the model to countries like the United States, where most households own multiple cars.
} 
$j$ to simplify notation), and the unit mass of cars in the economy is distributed among $\bar{a} \leq A$ age groups (vintages), where $A$ is an upper bound on the age of cars of any make/model due to their physical breakdown.

Following the literature, we assume that the net idiosyncratic component consumers' preferences are characterized by a common quasi-liner utility function $u(a)-\mu$ (trade costs) where the first term captures the utility of owning a car of age $a$ and the second term accounts for the monetary costs of trade. Trade costs consist of the difference in prices of traded cars, with the addition of transaction costs (to be specified below). The parameter $\mu>0$ is a simple way to capture income/wealth effects in this model. High values of $\mu$ can be interpreted as "being poor" because the cost of buying a new car will involve a high opportunity cost in terms of forgone consumption of other goods. Thus, $\mu$ will constitute a crucial parameter governing a consumer's willingness to pay for a car. A non-increasing function $u(a)$ captures the utility of from driving (utilization) as well as the expected cost of maintaining a car of age $a$. We assume that $u(a+1) \leq u(a)$ for $a \in 0, \ldots, A$.

Let transactions cost be given by an arbitrary function $T(P, a, d)$, dependent on the existing car of age $a$ to be traded for a different car of age $d$. For example, a combination of proportional transactions costs on the net trade $\rho \geq 0$ and a lump sum fee $T$ leads to a specification $T(P, d, a)=T+\rho[P(d)-P(a)]$. We assume that transactions costs are borne by the buyer, including sales or new cars where transaction cost also includes taxes charged by the government.

The state of a consumer is given by the vector $(a, \varepsilon)$ where $a \in\{1, \ldots, \bar{a}\}$ denotes the age of the current car that the consumer owns and $a=\varnothing$ if a consumer does not currently own a car. The cars in the model age deterministically, but in addition to normal aging with probability $\alpha(a) \in[0,1)$ a car may be involved in a stochastic accident that results in a total loss and subsequent scrappage. We model this event as a stochastic transition to the scrappage age $\bar{a}$.

The random component $\varepsilon$ is a vector with the number of elements equal to the number of state dependent discrete choices a consumer has. It captures idiosyncratic time-varying phenomena such as maintenance expenditures, search costs, and variability in the prices of traded cars that reflect idiosyncratic features of used cars that the buyer and seller observe that cause transaction prices to deviate from the average price $P(a)$. We assume that $\varepsilon$ has a multivariate extreme value distribution and that its elements are identically distributed and independent across choices, consumers, and time periods.

We assume that at the start of each period a consumer who owns a car of age $a$ can choose 
whether to trade or keep their car, which we denote by $d \in D(a)$ where $D(a)$ is a set of feasible trading choices we describe below. We assume trades take place instantaneously, so the car each consumer chooses is the one that is utilized during that period, and it might experience an accident during that time. At the start of the next period the chosen car is one year older, or in case of a major accident its condition transits to $\bar{a}$ since it is no longer drivable and must be scrapped. With this timing convention, it is impossible to start the period with a brand new car, so the state variable $a$ only takes values in $\{\varnothing, 1, \ldots, \bar{a}\}$. However consumers can trade their old car for a new one, so the set of feasible choices is $\{0, \ldots, \bar{a}-1\}$ which includes new cars. In addition, it is always possible to choose the outside option $d=\varnothing$ of having no car at all.

The set of feasible choices for a consumer whose car state is $a$ is thereby summarized as follows. If the consumer has no $\operatorname{car}(a=\varnothing)$ they can choose to remain in the no-car state $(d=\varnothing)$, buy a new car $(d=0)$, or one of the ages of cars available for sale in the secondary market, so $D(\varnothing)=\{\varnothing, 0,1, \ldots, \bar{a}-1\}$. If the consumer already owns a car of age $a<\bar{a}$, they have the option of keeping it $(d=\kappa)$ so $D(a)=\{\kappa, \varnothing, 0,1, \ldots, \bar{a}-1\}$. However, once a car reaches the scrappage age $\bar{a}$ we assume the consumer cannot keep their car so that $d=\kappa$ is eliminated from the choice set, i.e. $D(\bar{a})=\{\varnothing, 0,1, \ldots, \bar{a}-1\}=D(\varnothing)$.

Let $V(a, \varepsilon)$ be the value function for a consumer in state $(a, \varepsilon), a \in\{\varnothing, 1, \ldots, \bar{a}\}$. The Bellman equation for a consumer who does not own a car is

$$
\begin{aligned}
V(\varnothing, \varepsilon) & =\max \left[v(\varnothing, \varnothing)+\varepsilon(\varnothing), \max _{d \in\{0,1, \ldots, \bar{a}-1\}}[v(\varnothing, d)+\varepsilon(d)]\right], \\
v(\varnothing, d) & =u(d)-\mu[P(d)+T(P, d, \varnothing)]+\beta(1-\alpha(d)) E V(d+1)+\beta \alpha(d) E V(\bar{a}), \\
v(\varnothing, \varnothing) & =u(\varnothing)+\beta E V(\varnothing),
\end{aligned}
$$

where $\varepsilon(\cdot)$ denotes an element of the vector $\varepsilon$, and $E V(a)$ is the conditional expectation of the value function $V(a, \varepsilon)$ for a consumer whose car state is $a$. Similarly, the Bellman equation for a consumer who owns a car of age $a$ is given by:

$$
\begin{aligned}
V(a, \varepsilon) & =\max \left[v(a, \varnothing)+\varepsilon(\varnothing), v(a, \kappa)+\varepsilon(\kappa), \max _{d \in\{0,1, \ldots, \bar{a}-1\}}[v(a, d)+\varepsilon(d)]\right] \\
v(a, d) & =u(d)-\mu[P(d)-P(a)+T(P, d, a)]+\beta(1-\alpha(d)) E V(d+1)+\beta \alpha(d) E V(\bar{a}), \\
v(a, \kappa) & =u(a)+\beta(1-\alpha(a)) E V(a+1)+\beta \alpha(a) E V(\bar{a}), \\
v(a, \varnothing) & =u(\varnothing)+\mu P(a)+\beta E V(\varnothing),
\end{aligned}
$$


where $d=\kappa$ denotes the decision to keep the existing car $a$, which is different from the decision $d=a$ because in the latter case the transaction cost has to be paid. We assumed above that this option is not available when $a=\bar{a}$. The owner of car $a$ also has the option $d=\varnothing$ to sell the current car and move to the outside good state.

When $\varepsilon$ is distributed as IID Type I extreme value with mean normalized to zero and scale parameter $\sigma$, we can use the max-stability property of the extreme value distribution (see (McFadden, 1981)) to derive the well-known "log-sum" expressions for $E V(a) \equiv \int V(a, \varepsilon) d F(\varepsilon)$, $a \in\{1, \ldots, \bar{a}-1\}$ in equations (1) and (2) to get:

$$
E V(a)=\sigma \log \left(\sum_{d \in D(a)} \exp \{v(a, d) / \sigma\}\right)
$$

where the sum over feasible state-dependent choices $D(a)$ in equation (3) reflects the constraints that consumers in state $a=\{\varnothing, \bar{a}\}$ are not allowed to keep.

We can stack equations (3) into a system of nonlinear equations for the $(\bar{a}+1) \times 1$ vector of expected value functions $E V=(E V(\varnothing), E V(1), \ldots, E V(\bar{a}))$ as

$$
E V=\Gamma(E V, P)
$$

where $\Gamma$ is the smoothed Bellman operator. Rust $(1987,1994)$ shows that $\Gamma$ is a contraction mapping in $E V$ and hence has a unique fixed point $E V(P)$ for each value of $P .{ }^{9}$

The excess demand function $E D(P)$ in the model is defined in terms of choice probabilities, where $\Pi(d \mid a, P)$ is the probability that a consumer in state $a$ chooses option $d$ from the set of available options given the prices $P(a)$. Under the extreme value assumption for $F(\varepsilon)$ it is well known that $\Pi(d \mid a, P)$ is given by the standard multinomial logit formulas, which we can write as functions of $E V(a)$ defined by (3):

$$
\Pi(d \mid a, P)=\exp \{[v(a, d, P)-E V(a, P)] / \sigma\}
$$

where we include $P$ in the notation for $v(a, d, P)$ and $E V(a, P)$ to emphasize that these functions depend on prices $P(a), a \in\{0, \ldots, \bar{a}\}$.

\footnotetext{
${ }^{9}$ Note that using (1)-(2) and (3) we could formulate an equivalent fixed point problem in terms of choice specific functions $v(a, d)$. However, working with $E V(a)$ has a huge computational advantage in our case, because the fixed point has to be found in $\mathbb{R}^{\bar{a}+1}$ space rather than in $\mathbb{R}^{(\bar{a}+1)(\bar{a}+2)}$.
} 


\subsection{Equilibrium with idiosyncratic consumer heterogeneity}

Let $0 \leq q(a) \leq 1$ denote the fraction of cars of age $a$ in the economy such that the (row) vector $q \in \mathbb{R}^{\bar{a}}$ represents the age distribution of the car fleet. Following (Rust, 1985a) we refer to $q$ as the holdings distribution and it summarizes the distribution of cars at the start of the period before trading. Thus, new cars have zero mass in this distribution: though new cars may have been bought in the previous period, none will be brand new at the start of this period.

To account for the outside good we introduce the notation $p$ to denote the ownership distribution, whose first $\bar{a}$ components provide the fraction of the population who owns cars of ages $\{1, \ldots, \bar{a}\}$ and the last component provides the fraction of the population, $q(\varnothing)$, that has no car:

$$
p=(q[1-q(\varnothing)], q(\varnothing)) \in \mathbb{R}^{\bar{a}+1}
$$

In a stationarity equilibrium, both $q$ and $p$ will be time invariant. To be able to derive the equilibrium conditions in convenient matrix form we combine the choice probabilities from (5) into two $(\bar{a}+1) \times(\bar{a}+1)$ matrices, $\Delta^{T}(P)$ and $\Delta^{K}(P)$, for transitions due to cars that are traded and kept, respectively. The two matrices are defined as follows:

$$
\Delta^{T}(P)=\left[\begin{array}{cccccc}
\Pi(1 \mid 1, P) & \Pi(2 \mid 1, P) & \ldots & \Pi(\bar{a}-1 \mid 1, P) & \Pi(0 \mid 1, P) & \Pi(\varnothing \mid 1, P) \\
\Pi(1 \mid 2, P) & \Pi(2 \mid 2, P) & \ldots & \Pi(\bar{a}-1 \mid 2, P) & \Pi(0 \mid 2, P) & \Pi(\varnothing \mid 2, P) \\
\vdots & \vdots & & \vdots & \vdots & \vdots \\
\Pi(1 \mid \bar{a}, P) & \Pi(2 \mid \bar{a}, P) & \ldots & \Pi(\bar{a}-1 \mid \bar{a}, P) & \Pi(0 \mid \bar{a}, P) & \Pi(\varnothing \mid \bar{a}, P) \\
\Pi(1 \mid \varnothing, P) & \Pi(2 \mid \varnothing, P) & \ldots & \Pi(\bar{a}-1 \mid \varnothing, P) & \Pi(0 \mid \varnothing, P) & 0
\end{array}\right]
$$

and

$$
\Delta^{K}(P)=\operatorname{diag}(\Pi(\kappa \mid 1, P), \ldots, \Pi(\kappa \mid \bar{a}-1, P), 0, \Pi(\varnothing \mid \varnothing, P)),
$$

where $\operatorname{diag}(\cdot)$ denotes a diagonal matrix with given values on the main diagonal. Note that column $\bar{a}$ of $\Delta^{T}(P)$ does not represent the probability of buying a car of age $\bar{a}$, which is zero, since cars of this age can only be scrapped. Instead, this column is the probability of buying a new car: the reasons for doing this will become evident below.

Let $\Delta(P) \equiv \Delta^{T}(P)+\Delta^{K}(P)$ combine the trading and holding behavior transitions. By construction $\Delta(P)$ reflects the choice restrictions at each state $a$ introduced above, and forms a stochastic matrix which rows add up to 1 . We refer to $\Delta(P)$ as the trade transition probability 
matrix. Given the distribution of consumers $p$ at the start of the period, the matrix product $p \Delta^{T}(P)$ represents the distribution of consumers after instantaneous trading has taken place.

The matrices (7) and (8), allow us to express supply $S(P) \in \mathbb{R}^{\bar{a}-1}$ and demand $D(P) \in \mathbb{R}^{\bar{a}-1}$ for cars of ages $a \in\{1, \ldots, \bar{a}-1\}$, and thus excess demand $E D(P)=D(P)-S(P)$. Given our assumption of infinitely elastic demand for cars as scrap and infinitely elastic supply of new cars, there are only $\bar{a}-1$ free prices to clear the markets. Given the ownership distribution, $p$, supply and demand are defined by:

$$
\begin{aligned}
& p \Delta^{T}(P)=(D(P), \quad D(0, P), \quad D(\varnothing, P)), \\
& p\left(I-\Delta^{K}(P)\right)=\left(\begin{array}{lll}
S(P), & S(\bar{a}, P), & S(\emptyset, P)
\end{array}\right),
\end{aligned}
$$

where $I$ is the identity matrix of the appropriate size and $D(0, P), D(\varnothing, P), S(\bar{a}, P)$, and $S(\varnothing, P)$ are four scalars which denote respectively the fraction of consumers who choose to buy a new car, the fraction of consumers who choose not to have a car, the fraction of owners who scrap cars, and the fraction of consumers without a car who wish to buy one. Thus, excess demand is given by

$$
p(\Delta(P)-I)=(E D(P), \quad D(0, P)-S(\bar{a}, P), \quad D(\varnothing, P)-S(\varnothing, P)) .
$$

The right hand side of (10) is an $1 \times(\bar{a}+1)$ vector, whose first $\bar{a}-1$ components are the excess demand of cars of ages $a \in\{1, \ldots, \bar{a}-1\}$. The last two components equal the difference in the fraction of new and scrapped cars, and the difference in the fraction of consumers choosing the outside good, respectively. In a stationary equilibrium, the market clearing condition, $E D(P)=$ $(0, \ldots, 0)$, holds, but the latter two differences must equal zero as well.

Note that the condition $D(\varnothing, P)-S(\varnothing, P)=0$ identifies the fraction of consumers $q(\varnothing)$ who choose not to have a car conditional on the holdings distribution $q$. Combining (6) with (7) and (8), we get the following linear equation for $q(\varnothing, P)$ :

$$
[1-q(\varnothing, P)]\left[\sum_{a=1}^{\bar{a}} \Pi(\varnothing \mid a, P) q(a)\right]-q(\varnothing, P) \Pi(\varnothing \mid \varnothing, P)=0
$$

In addition to the ownership dynamics captured by transition matrix $\Delta(P)$, cars age and may get involved in accidents. The stationary equilibrium also has to account for the physical evolution of the car fleet. Dynamics of the car age distribution $q$ are governed in the model 
by the $\bar{a} \times \bar{a}$ Markov transition matrix $Q$ which we refer to as physical transition matrix. It reflects the deterministic aging of cars as well as stochastic accidents that manifest themselves as transitions to the terminal age $\bar{a}$, and subsequent scrappage:

$$
Q=\left[\begin{array}{cccccc}
0 & 1-\alpha(1) & 0 & \cdots & 0 & \alpha(1) \\
0 & 0 & 1-\alpha(2) & \cdots & 0 & \alpha(2) \\
\vdots & \vdots & \vdots & \ddots & \vdots & \vdots \\
0 & 0 & 0 & \cdots & 1-\alpha(\bar{a}-2) & \alpha(\bar{a}-2) \\
0 & 0 & 0 & \cdots & 0 & 1 \\
1-\alpha(0) & 0 & 0 & \cdots & 0 & \alpha(0)
\end{array}\right]
$$

The typical element of transition matrix $Q$ denoted $q_{i j}$ is interpreted as the probability of a car age $i$ to become age $j$ in the next period, independent of whether it was traded. Therefore, given the holdings distribution $q$ in one period, $q Q$ is the distribution of ages of all cars in the economy in the beginning of the following period, irrespective of the change of the ownership of these cars during current period trade.

Recall our convention that the holding distribution $q$ is the distribution of car ages at the start of the period before the trading occurs. When trade occurs, if the economy is in flow equilibrium, the fraction of cars that are scrapped will equal the fraction of new car sales. Thus, from the point of view of age transitions, all cars of age $\bar{a}$ "reappear" next period as new cars. This explains the non-zero first element in the last row of matrix $Q$ in (12). The cars entering the period as age $\bar{a}$ are replaced during the trading phase, and therefore experience accidents during the period with probability $\alpha(0)$. Owners do not have the option of keeping cars of age $\bar{a}$, so they sell them for their scrap value $\underline{P}$, and return to the market to buy a replacement car (or choose the outside good). The stationary equilibrium condition $D(0, P)-S(\bar{a}, P)=0$ discussed above ensures flow equilibrium, so that the overall stock of cars does not change over time.

However in a stationary equilibrium the entire holdings distribution cannot change over time, so we must have $q Q=q$. When $\alpha(a)=0$ for all ages, it is easy to see that the holdings distribution is uniform, with $q(a)=1 / \bar{a}, a \in\{1, \ldots, \bar{a}\}$. With $\alpha(a)>0$ for at least some ages, there is a unique time invariant distribution $q$ compatible with transition probability $Q$. In both cases, the following formulas give the analytic solution for the stationary holdings distribution 
for $a \in\{1, \ldots, \bar{a}-1\}$ :

$$
q(a)=q(\bar{a}) \prod_{i=0}^{a-1}(1-\alpha(i)), \quad q(\bar{a})=\left(1+\sum_{a=1}^{\bar{a}-1} \prod_{i=0}^{a-1}(1-\alpha(i))\right)^{-1}
$$

We are now in position to give a formal definition of equilibrium in the automobile market that extends the stationary equilibrium concept of Rust (1985b) to economies with positive transactions costs. This definition will form the basis for various extensions of the model that we will consider in subsequent sections of this paper.

Definition D1 (Stationary equilibrium in the automobile market). Stationary equilibrium in the market for new and used cars is given by the tuple $\{\bar{a}, P(a), q(P, \bar{a})\}$ such that the following conditions are satisfied:

(a) The price function $P(a)$ satisfies the infinite elasticity assumption for supply of new cars and demand of scrapped cars, so it holds $\underline{P} \leq P(a) \leq \bar{P}=P(0)$ for all $a \in\{1, \ldots, \bar{a}-1\}$, and $P(a)=\underline{P}$ for all $a \in\{\bar{a}, \ldots, A\}$;

(b) The stationary holdings distribution $q(P, \bar{a})$ constitutes a fixed point of the physical transition matrix $Q$, so it holds $q(P, \bar{a})=q(P, \bar{a}) Q$;

(c) Consumers follow their optimal trading strategies that follow from the solution of the dynamic problem (1)-(2);

(d) The market clearing conditions $E D(P)=(0, \ldots, 0)$ are satisfied for cars of ages between 1 and $\bar{a}-1$, as well as for the outside option, $D(\phi, P)=S(\phi, P)$, and the demand for new cars equals the supply of scrapped cars $D(0, P)=S(\bar{a}, P)$.

Given the simple relationship (6) between the holdings distribution $q$ and the ownership distribution $p$, it is convenient to introduce the equivalent of the physical transition matrix $Q$ for $p$. To reflect the natural constraint that a consumer who does not own a car once trading is over will also not own a car at the beginning of the next period, a constant 1 has to be added on the diagonal which leads to the following $(\bar{a}+1) \times(\bar{a}+1)$ block diagonal Markov transition matrix:

$$
\Omega=\operatorname{diag}(\mathrm{Q}, 1)
$$

Unlike $Q, \Omega$ is not irreducible and it is easy to see that the distribution of the form $(q r, 1-r)$ where $q$ is the fixed point of $Q$ and $r \in[0,1]$ is an invariant distribution of $\Omega$. 
Theorem 1 (Existence of equilibrium with idiosyncratic heterogeneity). The stationary equilibrium for the automobile economy with the idiosyncratically heterogeneous consumers defined in Definition D1, exists, and in equilibrium it holds

$$
p(P, \bar{a})=p(P, \bar{a}) \Delta(P)=p(P, \bar{a}) \Omega=p(P, \bar{a}) \Delta(P) \Omega,
$$

where the ownership distribution $p(P, \bar{a})$ is given by (6) and (11), and $\Omega$ is defined in (14).

Proof. Proof is provided in the Appendix B.1 (page 56).

The logic under the "double fixed point" characterization results of Theorem 1 is the following. Given (10), conditions (c)-(d) in D1 are satisfied if the ownership distribution is a fixed point of the trade transition matrix $\Delta(P)$. This is ensured by the first equality in (15). Stationarity of the holdings distribution (b) in D1 is given by the second equality of (15). The third equality is a simple substitution of one fixed point condition into the second, which has a straightforward interpretation as the steady state ownership distribution in the economy. The product of the two $(\bar{a}+1) \times(\bar{a}+1)$ Markov transition probability matrices $\Delta(P)$ and $\Omega$ is another Markov transition probability matrix that represents the evolution of the age distribution of cars in the economy. That is, for a given initial ownership distribution $p$ in the beginning of the period (where the first $\bar{a}$ components represent fractions of ownership of cars of age $a \in\{1, \ldots, \bar{a}\}$, and the last component represents the fraction of consumers without cars), the product $p \Delta(P)$ represents the distribution of holdings immediately after trading. Note that according to the structure of (7), the $\bar{a}$-th column of the matrix enforces the flow equilibrium restriction that scrapped cars are replaced by new cars, and therefore the $\bar{a}$-th element of $p \Delta(P)$ is the fraction of new cars. When we multiply this by the physical transition matrix $\Omega, p \Delta(P) \Omega$, we obtain the holdings distribution at start of the next period. Similarly, the structure of the trade transition matrix $\Delta(P)$ and the last column of the physical transition matrix $\Omega$ ensure that the last component of the holdings distribution represents the steady state fraction of consumers not owning a car.

Note that Theorem 1 only establishes the existence of the stationary equilibrium, but is silent about its uniqueness. In fact, our numerical exercises show that it is possible to find equilibrium price functions and holdings distributions with different values of $\bar{a}$, up to a certain level of scrappage age. With our assumption on non-increasing utility $u(a)$, there is an upper bound on the scrappage age $\bar{a}$ beyond which it becomes impossible to satisfy the condition 
(a) of Definition D1 with the price vector that satisfies (15). As discussed above, if cars are forced (by the matrix $Q$ ) to remain in the economy for too long, their owners have to be offered a premium in the form of "negative" price to compensate for the low level of utility from car utilization. We refer to the equilibrium with the highest $\bar{a}$ that satisfies Definition D1 as the maximal equilibrium. Based on our calculations, and the analogy with the homogeneous consumer economy case where we are able to provide analytic proofs in Appendix A, we form the following conjecture:

Conjecture J1. Out of all stationary equilibria in the automobile economy that satisfy Definition D1, the one with maximum scrappage age a achieves the maximum welfare.

\subsection{Computational algorithm}

Theorem 1 serves as a simple characterization of the stationary equilibrium as a solution to a system of non-linear equation given by (15). There are $\bar{a}$ independent equations (one is automatically satisfied because the elements of $p(P, \bar{a})$ add up to one), and $\bar{a}$ unknowns, namely $\bar{a}-1$ prices $P(a)$ for $a \in\{1, \ldots, \bar{a}-1\}$, and the share with the outside good $q(\varnothing, P)$, given by (11).

Our computational approach to solving for the stationary equilibrium is based on the iterative solution of system (15) equilibrium prices conditional on the scrappage age $\bar{a}$. The solution of the system is itself a nested loop computation, because for each trial value of price vector, the dynamic programming problem given by (1)-(2) has to be solved in order to form $\Delta(P)$. To ensure high computational performance, we apply Newton's method both on the inner loop for solving the dynamic programming problem, and the outer loop for solving for the equilibrium prices. The following lemma establishes the needed smoothness properties of the objects in the model:

Lemma L1 (Smoothness). The unique fixed point EV of the smoothed Bellman operator in (4), the choice-specific value functions $v(\cdot)$ in (1) and (2), the choice probabilities $\Pi(d \mid a, P)$ in (5), and trade transition probability matrix $\Delta(P)$ are continuously differentiable functions of $P$.

Proof. Proof is provided in the Appendix B.2 (page 56).

The smoothed Bellman equation (4) can be solved by successive approximation by repeated application of the operator $\Gamma$ to compute a series of approximations to the fixed point $E V(P)$ given by $E V_{k+1}=\Gamma\left(E V_{k}, P\right)$ for some initial $E V_{0}$. But for maximum computational efficiency 
we rely on the method of policy iteration (Howard, 1960) which is mathematically equivalent to the use of Newton's method to solve for $E V$ as a zero of the mapping $H(E V, P)=E V-$ $\Gamma(E V, P)=0$. It is easy to see from the definition of the smoothed Bellman operator $\Gamma$ that this is a continuously differentiable function of $E V$ so its gradient $\nabla_{E V} \Gamma(E V, P)$ exists and is a continuous function of $E V$ for any $P$. Then the policy iteration (Newton's method) algorithm provides a sequence of approximations $\left\{E V_{k}\right\}$ to the zero $H(E V, P)=0$ given by

$$
E V_{k+1}=E V_{k}-\left[I-\nabla_{E V} \Gamma\left(E V_{k}, P\right)\right]^{-1}\left[E V_{k}-\Gamma\left(E V_{k}, P\right)\right]
$$

Policy iteration typically converges after a small number of iterations to a highly accurate approximation of the true fixed point. In addition, the following lemma establishes a convenient analytic form for the derivative of the Bellman operator:

Lemma L2 (Derivative of Bellman operator). Let EV be the unique fixed point of the smoothed Bellman operator $\Gamma$ in (4), and let $\nabla_{E V} \Gamma(E V, P)$ be the Jacobian matrix of $\Gamma$ with respect to EV. The following holds:

$$
\nabla_{E V} \Gamma(E V, P)=\beta \Delta(P) \Omega
$$

where $\Delta(P)$ is the transition probability matrix given by the sum of (7) and (8), and $\Omega$ is the transition probability matrix given in equation (14).

Proof. Proof is provided in the Appendix B.3 (page 57).

The following algorithm finds the maximal stationary equilibrium:

1. Compute accurate starting values for the equilibrium search problem by solving for the homogeneous consumer economy equilibrium for the case of no transaction costs using the algorithm described in the Appendix A. ${ }^{10}$

2. Set $\bar{a}$ equal to the equilibrium scrappage age found in step 1 , and initialize the price vector at the computed equilibrium prices.

3. Solve for the stationary holdings distribution $q(\bar{a})$ as an invariant distribution of $Q$.

4. Using Newton's method, solve for the equilibrium prices as a solution to the system of non-linear equations given by (15). In particular, for each trial value of price vector:

(a) Solve the Bellman equation (4) for the fixed point EV using Newton's method;

\footnotetext{
${ }^{10}$ This is the limiting case with $\sigma \rightarrow 0$, and therefore provides excellent starting values for both prices and scrappage age. Conjecture J1 is also proven for that case, following Rust (1985b).
} 
Figure 1: Example stationary equilibrium

(a) Equilibrium prices

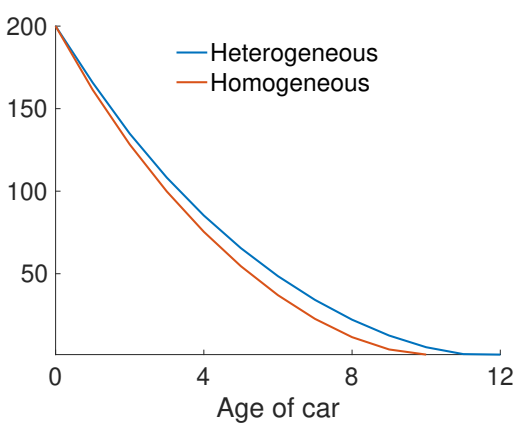

(b) Post-trade ownership distribution

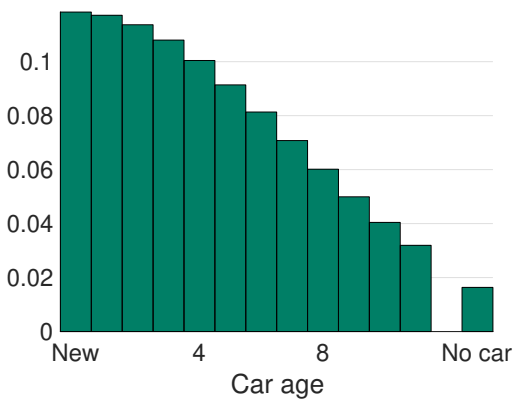

(c) Expected values of cars

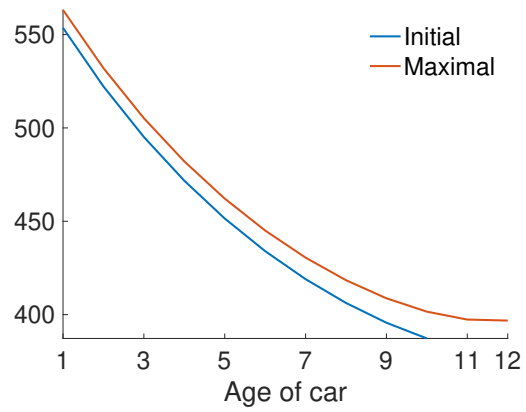

Notes: The optimal scrappage age in heterogeneous equilibrium is 12 years (10 in the homogeneous consumer economy). Post-trade ownership distribution in panel (b) is computed as $p \Delta(P)$.

(b) Form the trade transition matrix $\Delta(P)$ using (7)-(8), and compute $q(\varnothing)$ using (11);

(c) Compute the ownership distribution $p(P, \bar{a})$ using (6);

(d) Compute excess demand $E D(P)$ using (10);

5. If the resulting price vector which solves (15) satisfies condition (a) in Definition D1, increase $\bar{a}$ by one, and repeat step 3 .

6. If the resulting price vector which solves (15) does not satisfy condition (a) in Definition $\mathrm{D} 1$, decrease $\bar{a}$ by one, and repeat step 3.

7. Algorithm terminates when it finds such $\bar{a}$ that equilibrium condition (a) in Definition D1 are satisfied, but the same condition is not satisfied for $\bar{a}+1$. The maximal stationary equilibrium is then given by $\bar{a}$ as well as price function and the holdings distribution corresponding to this scrappage age.

\subsection{Illustrative example: Equilibrium trade with transactions cost}

The virtue of our computational approach is that we can compute equilibria for different $\bar{a}$ to confirm Conjecture J1. Figure 1 illustrates a computed maximal equilibrium for an example where $\bar{P}=200, \underline{P}=1, \beta=0.95, \sigma=5$ and $T(P, a, d)=1.5+0.03 P(d)$. Preferences are given by the utility function $u(a)=60-5 a$, and the marginal utility of money is $\mu=1$. We compute the scrappage threshold and shadow prices from the social planner's problem described in Appendix A, as shown in panels (a) and (c) for comparison, and use this equilibrium as our initial guess for $\bar{a}$ and $P$. In the maximal equilibrium $\bar{a}=12$, whereas the initial guess is $\bar{a}=10$.

There are several points to notice from the example equilibrium in Figure 1. First the 
homogeneous no transaction cost economy equilibrium is definitely not irrelevant: we see from panel (a) that the shadow prices arising from the optimal stopping social planning problem (Rust, 1985b) provide a good initial guess for the prices in the heterogeneous agent maximal equilibrium with transactions costs.

Panel (b) presents the stationary ownership distribution measured right after the instantaneous trading phase, so we term it post-trade ownership distribution. It is computed as $p \Delta(P)$. Due to non-zero accident probability, namely $\alpha(a)=0.01+0.02 a$, the age distribution of cars has a realistic downward sloping shape. Given our normalization of the utility of the outside good $u(\varnothing)=0$, nearly the entire population owns a car in steady state. Panel (c) of Figure 1

plots $E V(a)$ for $a \in\{1, \ldots, \bar{a}\}$ to show the welfare gains in maximal equilibrium compared to the heterogeneous equilibrium computed with $\bar{a}=10$. The plots show a strict Pareto improvement. Even the small fraction of consumers without cars experience welfare gain because the $E V(\varnothing)$ goes up in this example by $2.5 \%$ as well.

Thus, even though transactions costs inhibit trade, the gains from trade resulting from consumer heterogeneity more than compensate for the dampening effect of transactions costs and cars actually live longer in the heterogeneous agent maximal equilibrium.

\section{Equilibrium with Persistent Consumer Heterogeneity}

The economy with idiosyncratically heterogeneous consumers analyzed in the previous section is sufficient to support trade in presence of transactions costs. However, to assess the full gains from trade between consumers and to analyze equilibrium sorting of cars between different types of consumers, we now extend the framework to allow for persistent consumer heterogeneity.

\subsection{Equilibrium with time invariant heterogeneity}

We introduce the symbol $\tau$ to denote consumers of different types and assume that the type of a consumer does not change over time. Assume there are a finite number of types $\tau \in\left\{1, \ldots, N_{\tau}\right\}$ where $f(\tau)$ denotes the fraction of consumers of type $\tau$. The structure of the consumer decision problem (1)-(2) is identical for all consumers, but due to differences in preferences, the solution of this problem becomes type specific. The differences in demands and supplies are reconciled in the market equilibrium, where under assumption of stationarity, consumers end 
up "specializing" in their holdings of different ages of automobiles, reflecting the difference in their underlying preferences.

Let $u_{\tau}(a)$ be the utility for owning a car of age $a$ by consumers of type $\tau .^{11}$ Solving the Bellman equations (1)-(2) separately for each type, we obtain the decision-specific value functions $v_{\tau}(a, d)$, expected value functions $E V_{\tau}(a)$, and choice probabilities $\Pi_{\tau}(d \mid a, P)$, for each consumer type $\tau$, for all states $a \in\{1, \ldots, \bar{a}, \varnothing\}$ and for all choices $d \in D(a)$ subject to the same choice restrictions as in the previous section.

For a given value of $\bar{a}$, consumer heterogeneity does not change the variety of cars traded on the market. Therefore, the holdings distribution of cars is still given by the $1 \times \bar{a}$ vector $q \in \mathbb{R}^{\bar{a}}$; as in the case without time invariant heterogeneity. The same applies for the physical transition matrix $Q$ defined in (12), and as before the stationarity of equilibrium requires the holdings distribution to be an invariant distribution with respect to aging and accidents, so $q=q Q$ in any stationary equilibrium.

However, the ownership distribution $p$ now has a completely different structure compared to (6) due to the sorting of different types of consumers into particular ages of the cars. Thus, each type has its own ownership distribution that we denote by $p_{\tau}$, and a corresponding holdings distribution $q_{\tau}$. Let $q_{\tau}(\varnothing)$ be the fraction of type $\tau$ consumers who choose not to have a car. Analogous to (6), the ownership distribution of type $\tau$ is given by:

$$
p_{\tau}=\left(q_{\tau}\left[1-q_{\tau}(\varnothing)\right], q_{\tau}(\varnothing)\right) \in \mathbb{R}^{\bar{a}+1}
$$

The overall ownership distribution in the heterogeneous consumer economy is given by:

$$
p=\left(f(1) p_{1}, \ldots, f\left(N_{\tau}\right) p_{N_{\tau}}\right) \in \mathbb{R}^{N_{\tau}(\bar{a}+1)} .
$$

Note that because $f(\tau), \tau \in\left\{1, \ldots, N_{\tau}\right\}$ is a well defined probability distribution, the vector $p$ is also a probability distribution.

Let $\Delta_{\tau}^{T}(P)$ and $\Delta_{\tau}^{K}(P)$ be the ownership transfer and keep transitions matrices (7)-(8) for a particular consumer type $\tau$. To form the overall trade transition probability matrix we stack

\footnotetext{
${ }^{11}$ Throughout this section, we denote the type-specific variables with the $\tau$ subscript.
} 
$\Delta_{\tau}^{T}(P)$ and $\Delta_{\tau}^{K}(P)$ vertically before adding them to obtain the trade transition matrix $\Delta(P)$ :

$$
\Delta(P)=\left[\begin{array}{c}
\Delta_{1}(P) \\
\vdots \\
\Delta_{N_{\tau}}(P)
\end{array}\right]=\left[\begin{array}{c}
\Delta_{1}^{T}(P) \\
\vdots \\
\Delta_{N_{\tau}}^{T}(P)
\end{array}\right]+\left[\begin{array}{c}
\Delta_{1}^{K}(P) \\
\vdots \\
\Delta_{N_{\tau}}^{K}(P)
\end{array}\right]
$$

It is straightforward to verify that excess demand in the secondary market $E D(P)$ and the outside good $D(\varnothing, P)-S(\varnothing, P)$, as well as the imbalance between the new and scrapped cars $D(0, P)-S(\bar{a}, P)$ are given by an equation equivalent to (10), where identity matrix $I$ is replaced by the $N_{\tau}(\bar{a}+1) \times(\bar{a}+1)$ matrix obtained by stacking $N_{\tau}$ identity matrices vertically:

$$
p\left(\Delta(P)-\left(\begin{array}{c}
I \\
\vdots \\
I
\end{array}\right)\right)=(E D(P), D(0, P)-S(\bar{a}, P), \quad D(\varnothing, P)-S(\varnothing, P)) .
$$

It is immediately clear from (21) that unlike in the idiosyncratic case, the equilibrium holdings distribution in the economy with time-invariant consumer heterogeneity does not constitute a fixed point of the trade transition matrix $\Delta(P)$ in equilibrium when the right hand side of $(21)$ only contains zeros.

Theorem 2 (Existence of equilibrium with time invariant heterogeneity). The stationary equilibrium for the automobile economy with time invariant heterogeneous consumers defined in $D 1$, exists, and the equilibrium holdings distribution $q(P, \bar{a})$ is given by

$$
q(P, \bar{a})=\sum_{\tau=1}^{N_{\tau}} q_{\tau}(P, \bar{a}) f(\tau)
$$

where $q_{\tau}(P, \bar{a})$ is an internal part of the type-specific owner distribution $p_{\tau}(P, \bar{a})$ as per (18). The latter constitutes the invariant distribution of the transition probability matrix $\Delta_{\tau}(P) \Omega$ defined in (14) and (20):

$$
p_{\tau}(P, \bar{a})=p_{\tau}(P, \bar{a}) \Delta_{\tau}(P) \Omega, \tau \in\left\{1, \ldots, N_{\tau}\right\}
$$

Proof. Proof is provided in the Appendix B.4 (page 57).

Note that it is generally the case that $q_{\tau}(P, \bar{a}) \neq q_{\tau}(P, \bar{a}) \Delta_{\tau}(P)$, even in stationary equilib- 
rium. For example, excess demand for a given consumer type $\tau$ is generally not zero, since there can be net trade between different types of consumers in the economy. The only requirement that equilibrium imposes is that aggregate demand (for all types) equals the aggregate supply for $a=1, \ldots, \bar{a}-1$. There may be a net excess demand or supply of cars by different types of consumers reflecting patterns of specialization and gains from trade across different types of consumers. For example, poor consumers may choose to specialize in holding older cars while rich consumers specialize in holding newer cars, as we illustrate below.

Our computational approach to solving for an equilibrium in the economy with time invariant heterogeneity differs only slightly from the algorithm laid out in Section 2. The key difference is the calculation of the holdings distribution $q(P, \bar{a})$ : without persistent heterogeneity it follows directly from the physical transition matrix $Q$ and does not depend on prices, whereas in the heterogeneous case it is computed from invariant distributions of $\Delta_{\tau}(P) \Omega$ for each type (per Theorem 2), and therefore depends on prices. The following lemma establishes the prerequisite smoothness properties of the $q_{\tau}(P, \bar{a})$ in $P$, which is crucial for the successful application of Newton's method for solve for equilibrium prices. It shows that $p_{\tau}(P, \bar{a})$ computed as a fixed point (23) is a well-defined implicit function of $P$ that is continuously differentiable in $P$. This is not immediately evident, since a straightforward application of the Implicit Function Theorem would involve writing $p_{\tau}(P, \bar{a})$ as a zero of the following system of equations:

$$
q_{\tau}(P, \bar{a})\left[I-\Delta_{\tau}(P) \Omega\right]=0
$$

However, since $\Delta_{\tau}(P) \Omega$ is a transition probability matrix, it is easy to see that a vector of ones satisfies the equation. Hence, the matrix $\left[I-\Delta_{\tau}(P) \Omega\right]$ is singular, which violates a key condition needed for the application of the Implicit Function Theorem.

Lemma L3 (Smooth invariant distribution). For all $\tau$ the unique invariant distribution of the transition probability matrix $\Delta_{\tau}(P) \Omega$ is a continuously differentiable function of $P$.

Proof. Proof is provided in the Appendix B.5 (page 57).

We do not explicitly spell out the solution algorithm for the heterogeneous consumer economy for space considerations. It is completely analogous to the algorithm in Section 2 except for the calculation of type specific components in step 4 of the algorithm, needed to construct the overall ownership distribution $p$ as per (18)-(19) and compute the excess demand $E D(P)$ as per (21). As mentioned above, this requires the computation of the type specific holdings 
distribution as a fixed point (23) for each trial vector of prices, thus step 3 of the algorithm in Section 2 has to be incorporated into step 4 as well. All other steps remain exactly the same.

\subsection{Equilibrium with time varying heterogeneity}

The intermediate case between the idiosyncratic heterogeneity in Section 2 and time-invariant heterogeneity considered above is also of interest. Here we introduce an additional exogenously evolving Markovian state variable which denotes a time varying "class" of consumers. It appears that this case is closer to the idiosyncratic rather than to the persistent heterogeneous setting. Therefore to avoid repetition we present it in brief.

To fix ideas, let the additional state variable $y$ denote consumer level of income which could enter the utility of owning a car $u(a, y)$, or could enter the marginal utility of money $\mu(y)$. We assume that $y$ takes values from a finite set $\{y, \ldots, \bar{y}\}$, and evolves as an exogenous Markov process. Let $\pi$ denote the invariant distribution of this process, and let $\pi(y)$ be the invariant probability for this variable to take value $y$.

We do not repeat the Bellman equations (1)-(2) to conserve space: it is straightforward to generalize them to account for potentially changing next period $y$ by additional integration over it. In general, income will affect the values of different choices in different states, and this will induce different choice probabilities. Consequently, we add an additional argument to the model entities that become dependent on $y$ choice specific values $v(a, y, d)$ and add subscript $y$ to income specific trade transition matrices $\Delta_{y}(P)$ and equilibrium holdings and ownership distributions $p_{y}(P)$ and $q_{y}(P)$ etc. The trade transition matrix $\Delta(P)$ and physical transition matrix $\Omega$ are exactly as in the time invariant setting, while the structure of the ownership and holdings distributions mirrors their time invariant counterparts with $\tau$ replaced by $y$ and $f(\tau)$ replaced by $\pi(y)$ :

$$
p=\left(\pi(\underline{y}) p_{\underline{y}}, \ldots, \pi(\bar{y}) p_{\bar{y}}\right), \quad p_{y}=\left(q_{y}\left[1-q_{y}(\varnothing)\right], q_{y}(\varnothing)\right) \in \mathbb{R}^{\bar{a}+1}
$$

Theorem 3 (Existence of equilibrium with time varying heterogeneity). The stationary equilibrium for the automobile economy with time varying heterogeneity among consumers defined in D1, exists, and the equilibrium holdings distribution $q(P, \bar{a})$ is given by:

$$
q(P, \bar{a})=\sum_{y} q_{y}(P, \bar{a}) \pi(y)
$$


where $q_{y}(P, \bar{a})$ is an internal part of the income specific owner distribution $p_{y}(P, \bar{a})$ as per (25), and is given by:

$$
p_{y}(P, \bar{a})=(q(P, \bar{a})[1-q(\phi)], q(\phi)) \Delta_{y}(P) \Omega,
$$

where $q(\phi)=\sum_{y} q_{y}(\phi) \pi(y)$ denotes the overall fraction of consumers without cars, and $\Omega$ is defined in (14).

Proof. Proof is provided in the Appendix B.6 (page 58).

Note that unlike in Theorem 2 the income specific ownership distribution $p_{y}(P, \bar{a})$ does not constitute an invariant distribution, but is instead simply computed from the overall holdings distribution $q(P, \bar{a})$ per (27). In other words, in a stationary equilibrium the "mixing" between consumers of different incomes generates a stationarity in overall holdings even though we see in every time period differences in the holdings of cars of rich and poor consumers. With time-invariant heterogeneity instead, a consumer who is type $\tau$ in one period remains a type $\tau$ consumer in the next period, and thus the relevant distribution of holdings for consumers of each type $\tau$ is the same in both periods, explaining its description as an invariant distribution of the relevant transition probability matrix. The same does not happen here.

Note further that if instead of stacked $\Delta_{y}(P)$ as per (20) the trade transition matrix was defined by $\Delta(P)=\sum_{y} \Delta_{y}(P) \pi(y)$, the combination of (26) and (27) would lead to the "double fixed point" result as in Theorem 1. Thus, the case of time varying heterogeneity is equivalent to the case of idiosyncratic heterogeneous consumers once the trade transition matrices, as well as the holding and ownership distributions, are averaged over with invariant income transition probabilities. The computational algorithm for the stationary equilibrium in the economy with time varying heterogeneity is therefore the same as in Section 2.

Based on the analogy to the idiosyncratic case, further extension of the framework that would allow for the combination of time-invariant and time varying heterogeneity is therefore also possible. It is also clear that the dynamics of time varying heterogeneity could then be made type-specific without any additional cost.

\subsection{Illustrative example: Gains from trade and equilibrium sorting}

Figure 2 illustrates some of the equilibria in a heterogeneous agent economy with two types of consumers. The difference between this heterogeneous example and the example presented in Section 2.4 is the addition of a second consumer type with the same discount factor and utility 
Figure 2: Equilibrium price functions in a two consumer type economy

(a) Normal transaction costs

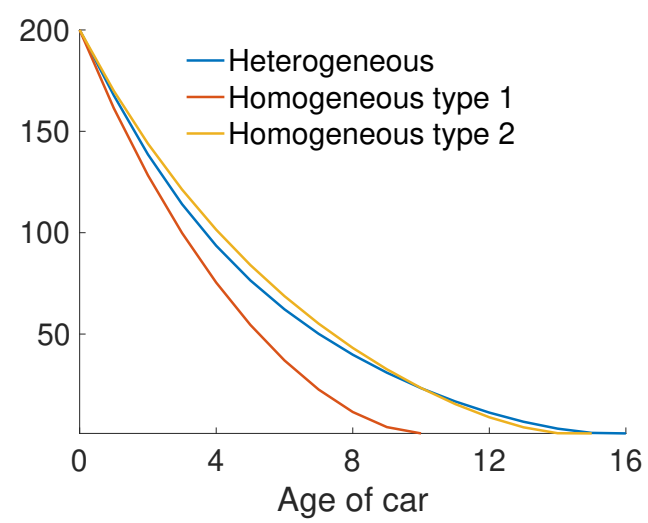

(b) High transaction costs

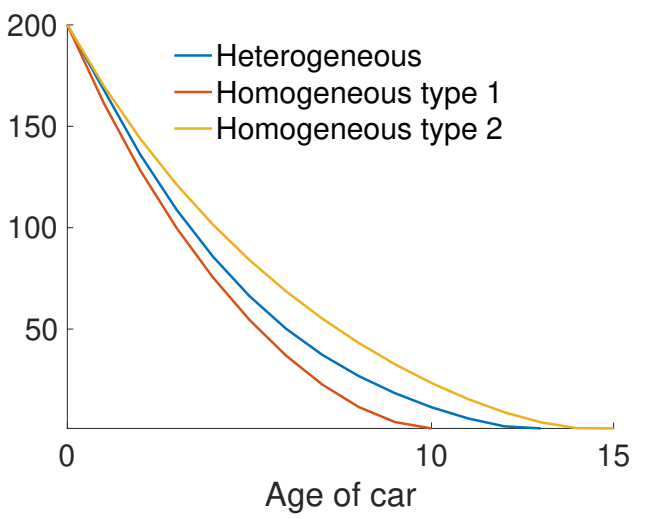

Notes: Both panels show the equilibrium price functions of the heterogeneous agent economy as well as homogeneous economies without transaction costs where all consumers are of either type. The heterogeneous equilibrium scrappage age is 16 in panel (a) and 13 in panel (b).

function $u(a)$, but different in their marginal utility of money, in that $\mu_{2}=1.75>1.0=\mu_{1}$. Thus, the first type of consumers who have a lower marginal utility of money $\mu_{1}=1$ are the rich consumer in the economy, while the second type are poor consumers. The utility of the outside good is set to 0 for both types. The heterogeneous agent equilibrium has $50 \%$ rich consumers and $50 \%$ poor consumers.

Thus it seems reasonable to conjecture that in equilibrium the fist type of consumers would be more likely to own newer more expensive cars. However it is not clear a priori what relative fractions of the two types of consumers will select into what fractions of the new and used cars, and which fraction choose to have no car at all. Therefore these questions as well as how transaction costs influences these distributions are of interest and can be answered by equilibrium calculation.

Figure 2 plots two equilibrium prices: one for the case where transactions costs are held at the same level as in example in Section 2.4, and the other for a much higher transaction cost, namely where the intercept of the cost function is increased to from 1.5 to 10 . Both panes (a) and (b) plot the heterogeneous agent equilibrium prices together with homogeneous equilibria without transaction costs where the economy is populated by the consumers of each type. When transactions costs are low there is an equilibrium with $\bar{a}=16$, but when transaction costs are high the maximum supportable equilibrium has $\bar{a}=13$. Thus the higher transactions costs have succeeded in partially "killing off" the market for used cars. 
Figure 3: Equilibrium ownership in a two consumer type economy

(a) Normal transaction costs

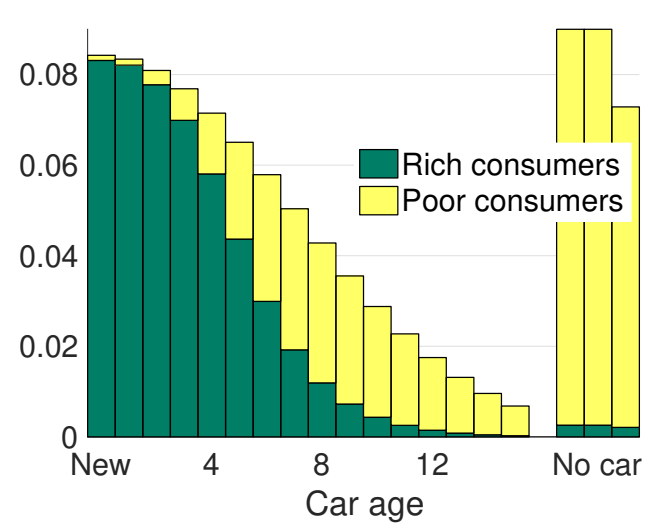

(b) High transaction costs

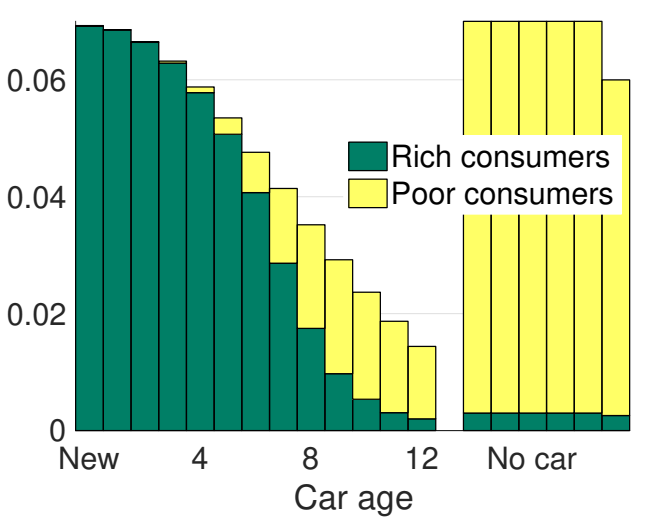

Notes: The total area of the bars in each panel adds up to one, presenting both the distribution of consumers over car ages, and the share of outside good. There are equal numbers of consumers of each type. Both panels show the post-trade ownership distributions. Since the density in the no-car state is much higher than each car-by-age category, we have split the no-car bar into several bars placed horizontally next to each other.

There is clear evidence of sorting of consumers into the ages of the car in panel (a) of Figure 3, which shows the post-trade ownership distribution. The rich consumers hold the newer cars and in particular are much more likely to buy new cars than the poor consumers. In addition, the fraction of poor consumers who do not to own a car is much higher. Overall, we see that poor consumers are driven out of the market to a much larger extent than the rich consumers when transactions cost increase.

These findings support specialization in holdings that enables gains from trade between the two types of consumers: the rich consumers buy brand new cars and hold them for several years and then sell them to poor consumers who also hold them for several years, trading the cars over a succession of poor owners until the car is scrapped. Thus, in this example the rich consumers are net suppliers of older cars, and the poor consumers are net demanders of older cars. Most of the trade between rich and poor consumers occurs for cars of roughly middle ages: the rich supply their middle aged cars to the poor consumers, and market clears in aggregate, but not type-by-type.

\section{Equilibrium with Multiple Automobile Makes/Models}

We now extend the equilibrium to $J>1$ different makes/models with corresponding new car prices $\bar{P}_{j}$, and scrap prices $\underline{P}_{j}, j=1, \ldots, J$. We continue to treat new car prices $\bar{P}_{j}$ as fixed, 
consistent with the small open economy framework. In Section 6 we endogenize new vehicle prices in a multiproduct oligopoly model of the new car market.

\subsection{Substitution patterns with multiple models of cars}

As emphasized by Berry, Levinsohn and Pakes (1995), the independence of the random preference shocks between choices can result in an undesirable pattern of equal cross-elasticities that are unrealistic in multi-product automobile markets. However many of these undesirable patterns are a consequence of a static choice model and would not hold in a dynamic model even under the multinomial logit specification for choice probabilities analyzed in the previous sections. We can accommodate even richer substitution patterns between different makes/models of cars via a generalized extreme value specification for idiosyncratic heterogeneity which leads to nested logit (NMNL) choice probabilities similar to Berkovec (1985) where we can control the variability and correlation of the random preference shocks to reflect natural patterns of similarity between different alternatives, such as similar unobserved attributes of different ages of the same make/model of car. This allows for a higher degree of persistence in choices for different makes/models of cars reflecting "brand loyalty." However we emphasize that a nested logit specification is not required to obtain brand loyalty: it can appear in the ordinary multinomial logit specification when consumers value one make/model of car sufficiently higher than others.

The pattern of correlation in the idioysncratic preference shocks in a NMNL specification can be illustrated by a "choice tree" where the upper level branches correspond to the choice of keeping the existing car $(j=\kappa)$, choosing the outside good $(j=\varnothing)$, or trading the existing car for another one. If the car is traded, correlation in preference shocks across different makes/models is reflected in a "subtree" with branches indexed by the different makes/models $j$. The lowest branches correspond to the choice of different car ages $a$ given a choice of a specific make/model $j$, as illustrated in Figure 4.

The NMNL choice tree results from the assumption that the multivariate distribution for the vector of idiosyncratic shocks to consumer utility $\varepsilon$ is a member of the generalized extreme value (GEV) family introduced by McFadden (1981). Each of the elements of vector $\varepsilon$ corresponds to the different discrete choices a consumer can make. The choice set for consumers who does not own a car is $D(\varnothing)=\left\{\varnothing,\left\{(j, d): j=1, \ldots, J, d=0, \ldots, \bar{a}_{j}-1\right\}\right\}$, where the choice $j=\varnothing$ corresponds to remaining in the no car state, and the choice $(j, d)$ corresponds to 
Figure 4: Choice trees for alternative nested logit specifications of the GEV distribution for $\varepsilon$

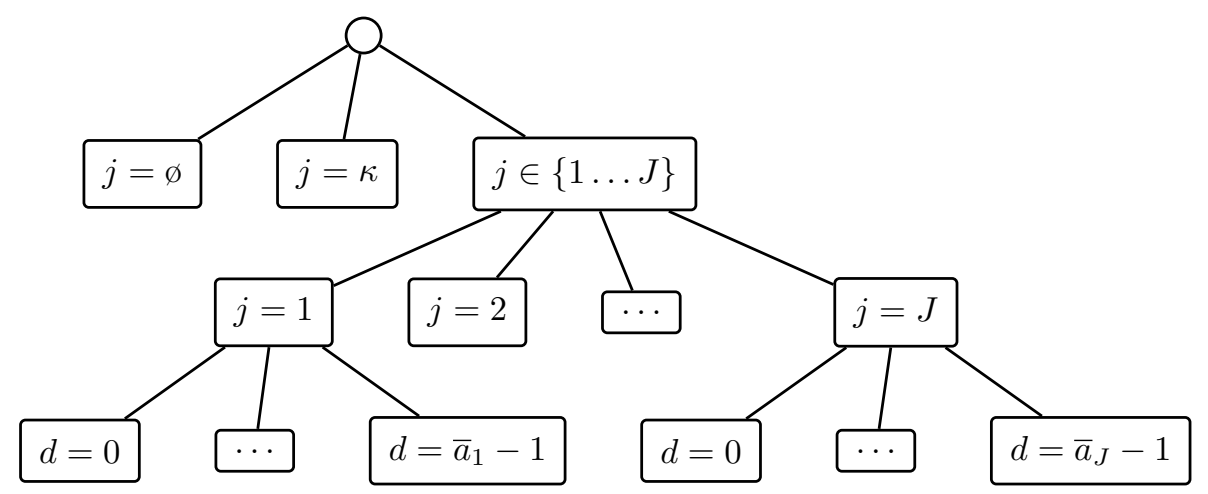

buying a car of type $j$ and age $d$. Similarly, the choice set for an individual who owns a car $(i, a)$ is given by $D(i, a)=\left\{\varnothing, \kappa,\left\{(j, d): j=1, \ldots, J, d=0, \ldots, \bar{a}_{j}-1\right\}\right\}$, where $j=\varnothing$ corresponds to the decision to purge their current car and own no car next period, $j=\kappa$ corresponds to the decision to keep their current car and the choice pair $(j, d)$ corresponds to the decision to trade their current car for another car of type $j$ and age $d$.

The joint GEV distribution of the shocks $\varepsilon$ is described in detail in Appendix D. The variability of choice at each nest of the nested decision tree in Figure 4 is controlled by the $J+2$ scale parameters $\sigma, \sigma_{j>0}$ and $\sigma_{j}$ correspondingly at the top level choice, intermediate level choice between car types, and the choice between new and used cars in each of the $J$ makes/models. By choosing any of the scale parameters to be sufficiently small, we can reduce the effects of random shocks in current utility on choices at each respective level of the decision tree. The additional $J+1$ parameter compared to the single car type setting we have considered so far, provide helpful flexibility in modeling complex substitution patterns among many ages and makes/models of cars. ${ }^{12}$ By equating the scales of random shocks at different levels of the decision tree, i.e. $\sigma=\sigma_{j>0}$, its layers collapse, and if $\sigma=\sigma_{j>0}=\sigma_{j}$ for all $j$, the nested choice specification reverts to the original MNL specification in Section 2.

Allowing for $J>1$ car types in the model introduces a new state variable $j$ interpreted as the make/model of the existing (in the beginning of a period) car. Consequently, the expressions for the value function and choice probabilities have to be adjusted to take this into account. Building on the notation of Section 3 we also allow for the multiple consumer types indexed

\footnotetext{
${ }^{12}$ The flexibility of the presented approach raises some concern about the identification of the scale parameters, as for example an alternative explanation with transaction costs can be offered to explain the dynamics of car switching behavior.
} 
with $\tau$. For the consumer who does not own a car we have the following Bellman equation:

$$
\begin{aligned}
V_{\tau}(\varnothing, \varepsilon)= & \max \left[v_{\tau}(\varnothing, \varnothing)+\varepsilon(\varnothing), \max _{j \in\{1, \ldots, J\}, d \in\left\{0,1, \ldots, \bar{a}_{j}-1\right\}}\left[v_{\tau}(\varnothing, j, d)+\varepsilon(j, d)\right]\right], \\
v_{\tau}(\varnothing, j, d)= & u_{\tau}(j, d)-\mu_{\tau}[P(j, d)+T(P, j, d, \varnothing)] \\
& +\beta(1-\alpha(j, d)) E V_{\tau}(j, d+1)+\beta \alpha(j, d) E V_{\tau}\left(j, \bar{a}_{j}\right), \\
v_{\tau}(\varnothing, \varnothing)= & u_{\tau}(\varnothing)+\beta E V_{\tau}(\varnothing),
\end{aligned}
$$

where $v_{\tau}(\varnothing, j, d)$ is the value of leaving the no car state to buy a car of type $j$ age $d, v_{\tau}(\varnothing, \varnothing)$ is the value of of remaining in the no car state, and an addition of index/argument $j$ signifies that the corresponding entity is car make/model specific. Similarly we extend the Bellman equation for the consumer who owns a car $(i, a)$ :

$$
\begin{aligned}
& V_{\tau}(i, a, \varepsilon)= \max \left[v_{\tau}(i, a, \varnothing)+\varepsilon(\varnothing), v_{\tau}(i, a, \kappa)+\varepsilon(\kappa),\right. \\
&\left.\max _{j \in\{1, \ldots, J\}, d \in\left\{0,1, \ldots, \bar{a}_{j}-1\right\}}\left[v_{\tau}(i, a, j, d)+\varepsilon(j, d)\right]\right], \\
& v_{\tau}(i, a, j, d)= u_{\tau}(j, d)-\mu_{\tau}[P(j, d)-P(i, a)+T(P, j, d, i, a)] \\
&+\beta(1-\alpha(j, d)) E V_{\tau}(j, d+1)+\beta \alpha(j, d) E V_{\tau}\left(j, \bar{a}_{j}\right), \\
& v_{\tau}(i, a, \kappa)= u_{\tau}(i, a)+\beta(1-\alpha(i, a)) E V_{\tau}(i, a+1)+\beta \alpha(i, a) E V_{\tau}\left(\bar{a}_{i}\right), \\
& v_{\tau}(i, a, \varnothing)= u_{\tau}(\varnothing)+\mu_{\tau} P(i, a)+\beta E V_{\tau}(\varnothing),
\end{aligned}
$$

where $v_{\tau}(i, a, \varnothing)$ is the value of purging one's current car of type $j$ age $a, v_{\tau}(i, a, \kappa)$ is the value of keeping the current car $(i, a)$, and $v_{\tau}(i, a, j, d)$ is the value of trading one's current car $(i, a)$ for another car $(j, d)$.

When $\varepsilon$ is a vector of GEV preference shocks, the expected value functions $E V_{\tau}(j, d)$ and the conditional choice probabilities have more complicated formulas than the ones given in equations (3) and (5). Computing the choice probabilities requires not only the choice specific values $v(\cdot, \cdot)$ but also the "inclusive values" equal to the expected maximum utility from the choice on the lower levels of the nested choice structure. However, the choice probabilities are still given by analytical expressions that can be computed directly using the formulas provided in full detail in Appendix D.

Even though $E V_{\tau}(j, d)$ has a more complicated form, all the contraction mapping theory applies to the dynamic programming problem set out in (28) and (29) in exactly the same way 
as before. Moreover, the results in Lemmas L1, L2 and L3 continue to apply, making possible the use of Newton's method for finding $E V_{\tau}$ as the fixed point of the Bellman operator (4) and the equilibrium prices - similar to the model with only one type of car.

\subsection{Stationary equilibrium in the market with multiple types of cars}

The introduction of the multiple car types into the model has surprisingly little effect on the equilibrium definition or computational algorithm, other than increasing the dimensionality of the problem. The results of Sections 2 and 3 apply with minimal adjustments after we redefine the holdings and ownership distributions $q$ and $p$, and correspondingly the trading and physical transition matrices $\Delta(P)$ and $\Omega$ for the case $J>1$.

With multiple types of cars $j=\in\{1, \ldots, J\}$, both the accident probability $\alpha(j, a)$, and more importantly the scrappage age $\bar{a}_{j}$ may vary by $j$. Thus, we have the $J$ secondary markets each trading $\bar{a}_{j}-1$ used cars of different ages. It is convenient to define the total number of car age-types as:

$$
\bar{a} \equiv \sum_{j=1}^{J} \bar{a}_{j},
$$

so that the total number of goods traded on the secondary market is $\bar{a}-J$. We let $P=$ $\left(P_{1}, P_{2}, \ldots, P_{J}\right) \in \mathbb{R}^{\bar{a}-J}$ be the concatenated vector of prices for all traded used cars in the economy that must be determined in a stationary equilibrium of the model.

Like prices, the holdings distribution of the cars of all makes/models is also a concatenated vector of the form:

$$
q=\left(q_{1} \eta_{1}, \ldots, q_{J} \eta_{J}\right) \in \mathbb{R}^{\bar{a}}
$$

where $q_{j} \in \mathbb{R}^{\bar{a}_{j}}$ gives the age distribution of cars of type $j$, and the elements of $\eta=\left(\eta_{1}, \ldots, \eta_{J}\right)$ are the market shares of the cars of different types. In stationary equilibrium for each $j$ the age distribution $q_{j}$ has to constitute an invariant distribution of the type-specific physical transition matrix $Q_{j}$ defined by (12) as before, i.e. $q_{j}=q_{j} Q_{j}$. The structure of the ownership distribution $p \in \mathbb{R}^{N_{\tau}(\bar{a}+1)}$ is inherited from the heterogeneous consumer model, so the formulas (18) and (19) continue to hold. In combination with (31), the type specific ownership distribution is given by:

$$
p_{\tau}=\left(\left(q_{\tau, 1} \eta_{1}, \ldots, q_{\tau, J} \eta_{J}\right)\left[1-q_{\tau}(\varnothing)\right], q_{\tau}(\varnothing)\right) \in \mathbb{R}^{\bar{a}+1}
$$

The extended physical transition matrix $\Omega$ as before describes the aging and stochastic 
scrappage of cars, as well as the trivial transition of the outside good, but has to incorporate all $J$ types of cars. It takes the following block-diagonal structure:

$$
\Omega=\operatorname{diag}\left\{Q_{1}, \ldots Q_{J}, 1\right\}
$$

where each of the car type-specific $\bar{a}_{j} \times \bar{a}_{j}$ transition matrices $Q_{j}$ is given by (12). For a holdings distribution $q$ at one time period, irrespective of changes in ownership, $q Q$ gives the holdings distribution in the following time period. It follows from the block-diagonal structure of $\Omega$ that (similar to the case with only one type of car) there is a continuum of invariant distributions satisfying $p \Omega=p$, however there are $J-1$ additional free parameters in constructing these invariant distributions. More precisely, any combination of the invariant distributions $q_{j}$ (such that $\left.q_{j}=q_{j} Q_{j}\right)$ of the form $\left(q_{1} \omega_{1}, \ldots, q_{J} \omega_{J}, 1-\sum_{j} \omega_{j}\right)$ constitutes an invariant distribution of $\Omega$ for the appropriately defined weights $\omega$. In equilibrium, these weights are jointly determined by the market shares $\eta_{j}$ as well as the share of the outside good $q(\varnothing)$.

To describe the market clearing conditions as well as the flow equilibrium conditions we follow the same scheme as in Section 3. In the presence of multiple car types, the structure of the trade transition matrix $\Delta(P)$ is still given by (20), but its consumer type-specific component $\Delta_{\tau}(P)$ has to be redefined as a $\bar{a}+1 \times \bar{a}+1$ transition matrix of the form:

$$
\Delta_{\tau}(P)=\left[\begin{array}{cccc}
\Delta_{\tau}(1 \mid 1, P) & \ldots & \Delta_{\tau}(J \mid 1, P) & \Delta_{\tau}(\varnothing \mid 1, P) \\
\vdots & \ddots & \vdots & \vdots \\
\Delta_{\tau}(1 \mid J, P) & \ldots & \Delta_{\tau}(J \mid J, P) & \Delta_{\tau}(\varnothing \mid J, P) \\
\Delta_{\tau}(1 \mid \varnothing, P) & \ldots & \Delta_{\tau}(J \mid \varnothing, P) & \Pi_{\tau}(\varnothing \mid \varnothing, P)
\end{array}\right]
$$

There are four types of components in (34): (a) square $\bar{a}_{j} \times \bar{a}_{j}$ matrices $\Delta_{\tau}(j \mid j, P)$ are the counterparts of the trade transition matrices $\Delta_{\tau}(P)=\Delta_{\tau}^{T}(P)+\Delta_{\tau}^{K}(P)$ given in (7)-(8) with the exception that the last row and last column corresponding to the outside option are dropped; (b) potentially non-square $\bar{a}_{i} \times \bar{a}_{j}$ matrices $\Delta_{\tau}(j \mid i, P)$ are composed of the type $i$ cars owners' choice probabilities $\Pi_{\tau}(j, d \mid i, a, P)$ to trade for cars of type $j$; (c) $1 \times \bar{a}_{j}$ row vectors $\Delta_{\tau}(j \mid \varnothing, P)$ are composed of choice probabilities of consumers who don't own a car to purchase type $j$ car, and (d) $\bar{a}_{i} \times 1$ column vectors $\Delta_{\tau}(\varnothing \mid i, P)$ are composed of choice probabilities of the owners of type $i$ cars to purge their cars.

With this notation, the market clearing conditions can be expressed using $\Delta_{\tau}(P)$ transition 
matrix similar to the way it is done in Sections 2 and 3. In particular, in the single consumer type case when $N_{\tau}=1$, the invariant distribution of $\Delta_{\tau}(P)$ (34) is market clearing distribution for given prices, which by Theorem 1 constitutes one of the conditions for the equilibrium in this case. When $N_{\tau}>1$, the results of Theorem 2 also hold with the $\Omega$ matrix and $\Delta_{\tau}(P)$ defined respectively by (33) and (34) above. When $N_{\tau}>1$, the analog of formulas (10) and (21) can easily be derived, but the right hand side is somewhat more complicated:

$$
p\left(\Delta(P)-\left(\begin{array}{c}
I \\
\vdots \\
I
\end{array}\right)\right)=\left(E D_{1}(P), \ldots, E D_{J}(P), D(\varnothing, P)-S(\varnothing, P)\right)
$$

where $E D_{j}(P)=\left(D_{j}(P)-S_{j}(P), D_{j}(0, P)-S_{j}\left(\bar{a}_{j}, P\right)\right)$ is the $1 \times \bar{a}_{j}$ vector of excess demands on the secondary market including the flow equilibrium condition $D_{j}(0, P)-S_{j}\left(\bar{a}_{j}, P\right)$ for car of type $j$, and $I$ denotes a $\bar{a}+1 \times \bar{a}+1$ identity matrix. With some matrix algebra it is possible to show using (32) that the flow equilibrium condition for each car type $j, D_{j}(0, P)=S_{j}\left(\bar{a}_{j}, P\right)$, can be expressed as

$$
0=\sum_{\tau=1}^{N_{\tau}} f(\tau)\left(\left[1-q_{\tau}(\varnothing)\right] \sum_{i=1}^{J} \eta_{i} \sum_{a=1}^{\bar{a}_{i}} q_{\tau, i}(a) \Pi(j, 0 \mid i, a, P)+q_{\tau}(\varnothing) \Pi(j, 0 \mid \varnothing, P)\right) .
$$

Equations (36) for $j \in\{1, \ldots, J\}$ form a linear system of $J$ equations that allow us to compute $J$ unknown market shares $\eta_{j}, j \in\{1, \ldots, J\}$, conditional on prices. Because the elements of (32) have to sum up to one, it follows that once market shares are determined, the share of the outside good for each consumer type $\tau$ can be found as a residual.

Theorem 4 (Existence of equilibrium with multiple car types). The stationary equilibrium for the automobile economy with multiple car types defined in D1, exists, and is given by:

(a) The conditions of Theorem 1 in the case of a single consumer type, $N_{\tau}=1$;

(b) The conditions of Theorem 2 in the case of time-invariant consumer heterogeneity; and

(c) The conditions of Theorem 3 in the case of time-variant consumer heterogeneity.

The required holdings and ownership distributions are given by (31) and (32), physical transition matrix is given by (33), and the trade transition matrix by (34).

Proof. Proof is provided in the Appendix B.7 (page 58).

The solution algorithm for the case of multiple car and consumer types is completely analo- 
Figure 5: Stationary equilibrium with 2 car types and 2 consumer types

(a) Equilibrium prices

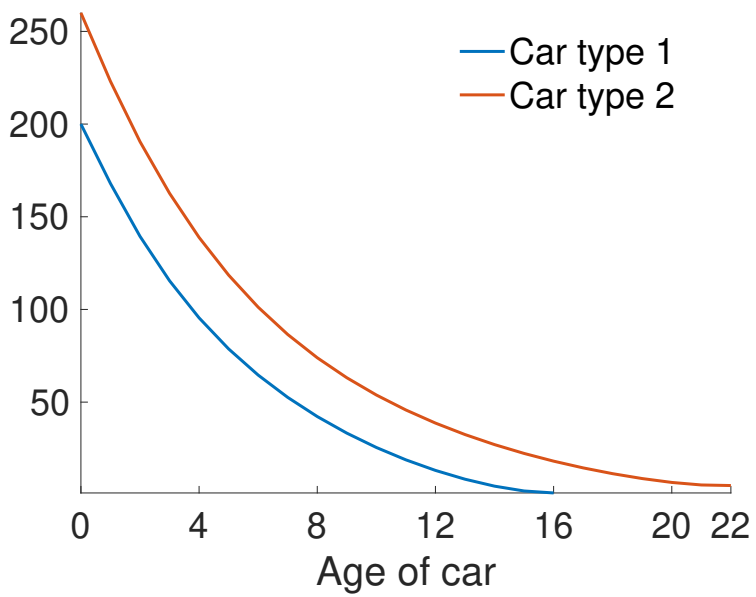

(b) Post-trade ownership distribution

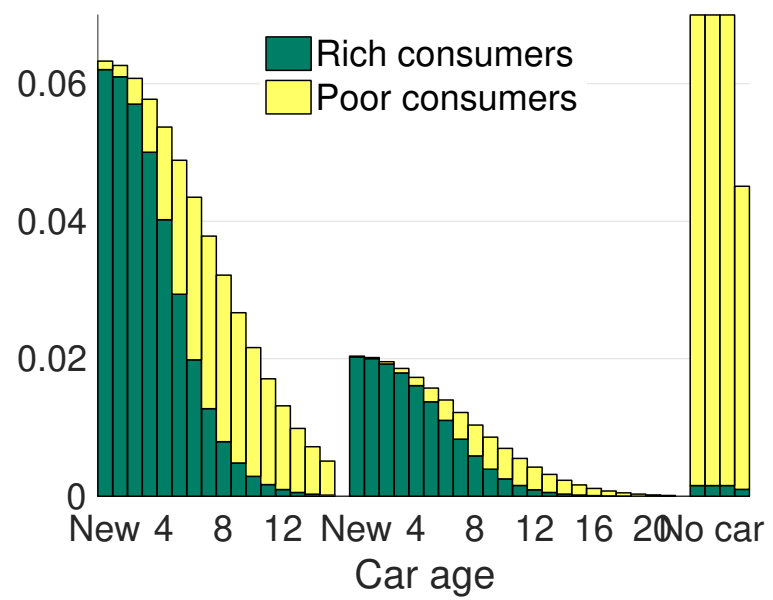

Notes: Panel (b) shows post-trade ownership distribution computed by $p \Delta(P)$ for each car type. The sum of the area of all bars adds up to one. Equilibrium scrappage age is 16 year for normal and 22 year for luxury car types.

gous to the algorithm in Section 3 with the redefined transition matrices as per Theorem 4, and an additional step to compute the market shares of different types of cars by solving a linear system (36). It is possible to show that the analogs of Lemmas L2 and L3 hold in the multiple car type case, and it is easy to see that (36) define the market shares as an implicit function of prices whose gradient can be calculated analytically. Hence, Newton's method can be used to solve for the fixed point of $\Gamma\left(E V_{\tau}\right)$ and for the zero of $E D(P)$ for the equilibrium prices $P$.

Similar to the single car case, we define a maximal equilibrium as the largest vector of scappage ages for the $J$ cars, $\left(\bar{a}_{1}, \ldots, \bar{a}_{J}\right)$ that support the price functions that satisfy the assumptions on infinite elastic supply of new cars and demand for scrap cars. The only adjustment to the algorithm from Section 2 for the multiple types of cars is that validity of equilibrium prices is checked one by one for all $j \in\{1, \ldots, J\}$ types of cars while keeping the scrappage ages of the other types fixed. When we are no longer able to individually increment the scrappage ages of any of the $J$ car types without violating the price constraint, then the algorithm terminates and returns the last estimate of the maximal equilibrium as the stationary equilibrium for the auto market. 


\subsection{Illustrative example: Equilibrium with two car models and two consumer types}

Figure 5 illustrates an example equilibrium with $J=2$ car types and 2 consumer types. The parameters of the consumer preferences and the first type of the car are the same as in Sections 2.4 and 3.3. The second car type in the economy is a luxury car with higher price $\bar{P}=260$ (30\% higher), higher scrap value $\underline{P}=5$. The luxury car is also more desirable for the consumers of both types, i.e. $u(a)=65-4.75 a$, while the normal car delivers the utility of $u(a)=60-5 a$. Both cars have the same transaction costs and accident probabilities.

We would expect that consumers not only sort themselves into newer and older cars by their marginal utility of money, but also own different types of cars in equilibrium. Indeed, in the computed maximal equilibrium we can see strong sorting in both types of cars and ages of cars, see panel (b) in Figure 5. The rich consumers mostly buy new cars of both types, and almost exclusively the new luxury cars. The holding behavior also differs by both consumer and car type. The rich consumers hold normal cars for about 5-7 years, and then sell them to a sequence of poor consumers who hold these car until considerable longer until scrappage. The luxury cars, even though their market share is significantly lower, are kept for much longer, and as a result, their equilibrium scrappage age is 22 years while for normal cars it is only 16 years.

\section{Analysis of Danish Car Tax Policy}

In this section we illustrate the use of the model for policy analysis. We conduct a stylized analysis of various tax reforms that have been under discussion in Denmark, which has one of the highest marginal tax rates on new vehicles in the world: $180 \%$ prior to 2016 and $150 \%$ after 2016 on all new vehicles whose price exceeds about $K=81,000$ DKK. The total tax revenue from cars, including registration taxes, annual fees, and fuel taxes, make up about approximately 4 to 7 percent of annual tax revenue, so it is important to have accurate predictions of the fiscal impacts of tax reforms. We consider a revenue-neutral counterfactual policy change that reduces marginal tax rates for the vehicle registration tax by $50 \%$ and increases fuel taxes to make up for the lost revenue. We extend our framework to also include driving decisions to illustrate its suitability for analyzing the effects of this policy change. This also allows us to study the environmental and congestion impacts of policy changes. 


\subsection{Utility specification: adding driving choice}

In this section we extend the model to endogenize driving, which is essential for analyzing how consumers would react to a substantial increase in the gas tax. Obviously, a main reason to have a car is to drive it and the development of our model so far has ignored this key aspect of a car. Let $x$ denote the number of kilometers a consumer chooses to travel in a period, and let $P_{j}^{x}$ denote the price per kilometer traveled for a car of type $j$. This equals the price of fuel (e.g. kroner per liter) divided by the car's fuel efficiency (kilometers per liter of fuel) for car type $j$.

Let $u_{\tau}(j, a, x)$ be the utility a consumer of type $\tau$ obtains from owning a car of type $j$ and age $a$ and driving it $x$ kilometers during the period. We assume that the probability of an accident and other physical deterioration in an automobile is independent of driving, $x$, and is instead only a function of car age $a$. The benefit of this rather strong assumption is that driving becomes a static sub-problem of the consumer's overall dynamic optimization problem. Without it, continuous driving choice has dynamic consequences, and the consumer must consider the effect of current driving on the future physical status of their car.

With this assumption, driving choices become independent of the trading decisions, and thus the optimal amount of driving $x_{\tau}\left(j, a, P_{j}^{x}\right)$ solves the static sub-problem of maximizing the driving utility net of monetary cost, $u_{\tau}(j, a, x)-\mu_{\tau} x P_{j}^{x}$. Substituting $x_{\tau}\left(j, a, P_{j}^{x}\right)$ back into the utility function $u_{\tau}(j, a, x)$ we obtain the indirect utility $u_{\tau}(j, a)$ for owning a car of age $a$ that incorporates the individual's optimal choice of driving. Assuming $P_{j}^{x}$ is time-invariant, the resulting model falls within the specification of Section 4.

To model the discrepancy between the theoretical optimal amount of driving and actual data on kilometers traveled by different cars between (bi-annual) inspections in Denmark, we treat $x_{\tau}\left(j, a, P_{j}^{x}\right)$ as planned driving. Actual driving responds to the unexpected needs for driving that cannot be predicted exactly. Consider the following utility specification that leads to a simple econometric model, which we estimate using Danish data:

$$
u_{\tau}(j, a, x)=(\gamma(j, \tau)+\xi) x-\frac{\phi}{2} x^{2}, \gamma(j, \tau)=\gamma_{j}+\gamma_{\tau}+\gamma_{1} a+\gamma_{2} a^{2}
$$

We assume that $\xi$ is a random variable that represents various unobserved needs for driving. Taking into account the cost of driving $\mu_{\tau} x P_{j}^{x}$, the optimal level of driving $x_{\tau}\left(j, a, P_{j}^{x}\right)$ implied 
by this structure is:

$$
\begin{aligned}
x_{\tau}\left(j, a, P_{j}^{x}\right) & =\frac{1}{\phi}\left[-\mu_{\tau} P_{j}^{x}+\gamma_{j}+\gamma_{\tau}+\gamma_{1} a+\gamma_{2} a^{2}+\xi\right] \\
& =-\beta_{\tau}^{P} P_{j}^{x}+\beta_{j}^{c a r}+\beta_{\tau}^{t y p e}+\beta^{a 1} a+\beta^{a 2} a^{2}+\xi .
\end{aligned}
$$

Using a simple linear regression we estimate coefficients of (38), and given the marginal utility of money $\mu_{\tau}$ compute the parameters of the utility function (37), to perform the counterfactual policy simulation. ${ }^{13}$

\subsection{Calibration and policy simulations}

To assess heterogeneous effects of the reform, we set up the model with $J=2$ car types and $N_{\tau}=2$ types of consumers, rich and poor, depending on whether their income is above or below the median. The two car types $j=\{1,2\}$ are defined by dividing the Danish car-fleet in two groups based on weight, where $j=1$ refers to a smaller, cheaper and slightly more fuel efficient car and $j=2$ refers to a larger, more durable and expensive car. We use the Danish micro data from Gillingham and Munk-Nielsen (2019) to estimate the reduced-form driving equation (38) to obtain coefficients: $\beta^{P}=(-2.6447,-5.9003), \beta^{\text {car }}=(0,3.0267), \beta^{\text {type }}=(20.134,20.818)$, $\beta^{a 1}=-0.19906$, and $\beta^{a 2}=0.0029431$. Given the reduced-form driving estimates, we then calibrated the marginal utility of money $\mu_{\tau}$ and an additional car type specific fixed effect in the utility function $u(j, a)$ to match the total fraction of cars, the fractions of new cars owned by consumers of different types, and the fraction of cars older that 15 years in the overall car age distribution. ${ }^{14}$

Using this specification we analyzed the effects of a revenue neutral reform that reduces registration tax rates for new vehicles by half and simultaneously increases the fuel tax rate by about $12 \%$ (from $100 \%$ to $112 \%$ ) to keep the policy change revenue neutral. ${ }^{15}$ The results of the counterfactual simulation are presented in Figure 6 and Table 1.

The top panels of Figure 6 illustrate the equilibrium prices and holdings under the tax sys-

\footnotetext{
${ }^{13}$ We ignore the restriction $x\left(j, a, P_{j}^{x}, \xi\right) \geq 0$ which implies that $\xi$ must be a truncated normal distribution. Also, because marginal utility of money $\mu_{\tau}$ differs by consumer type, technically all coefficients of the utility function (37) become type specific. For notation simplicity we do not add additional $\tau$ subscripts to reflect this dependence.

${ }^{14}$ Details on data and estimation results are available upon request.

${ }^{15}$ The registration taxes are given by a piecewise linear tax rule with two major brackets, such that new car prices after value added tax are given by $\bar{P}=\left(\bar{P}^{0}+T\left(\bar{P}^{0}\right)\right)$ where, $\bar{P}^{0}$ is the price of a new car including 25\% VAT but before other taxes, and $T\left(\bar{P}^{0}\right)$ is the vehicle registration tax $T\left(\bar{P}^{0}\right)=t_{1} \min \left(\bar{P}^{0}, K\right)+t_{2} \max \left(\bar{P}^{0}, 0\right)$. The top marginal tax rate, $t_{2}$, was reduced from 1.8 to 1.5 in 2016 , and $t_{1}$ was reduced from 1.05 to 0.85 in 2019 .
} 
Figure 6: Counterfactual simulation of automobile taxation reform in Denmark.

(a) Equilibrium prices (baseline)

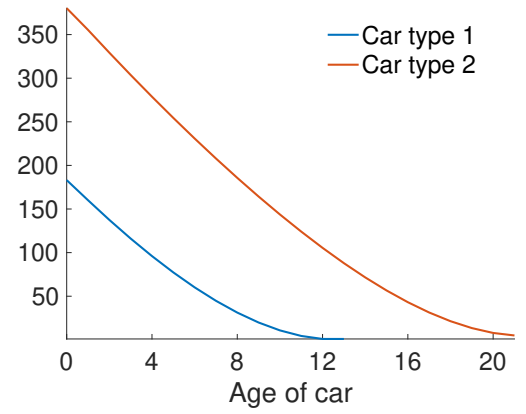

(d) Equilibrium prices (sim)

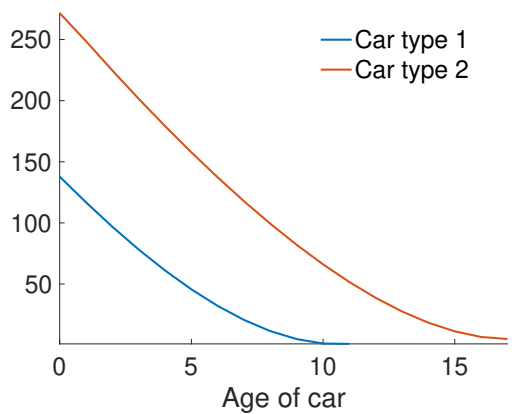

(b) Ownership (baseline)

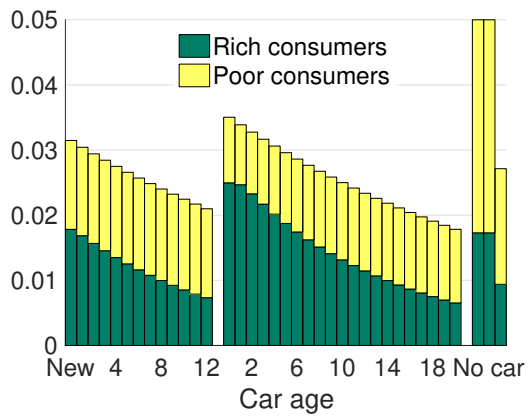

(e) Ownership (sim)

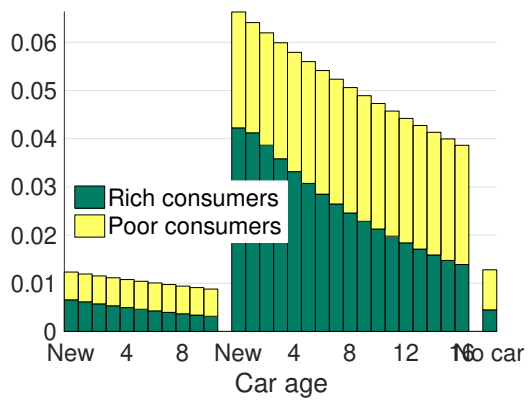

(c) Tax revenue (baseline)

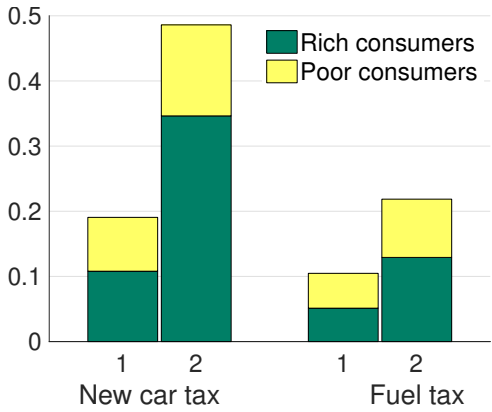

(f) Tax revenue (sim)

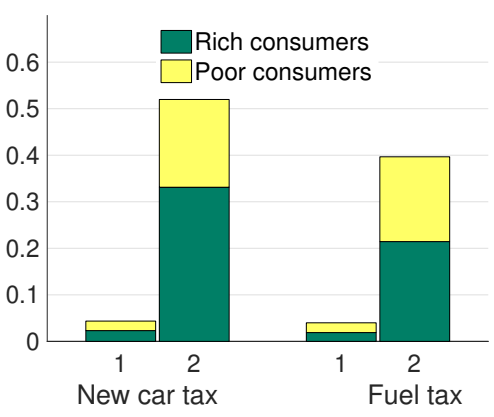

Notes: Panels (a) to (c) show the base line economy, panels (d) to (f) show the counterfactual equilibrium with taxes on new cars reduced by half, but the tax on fuel increased from about 100\% to $112 \%$. The equilibrium scrappage ages are 13 and 21 in panel (a), and 11 and 17 in panel (e). Panels (b) and (e) plot post-trade ownership distributions. Panels (c) and (f) display the composition of tax revenue across car types (1 and 2), consumer types and two sources (new car tax and fuel tax).

tem with up to $180 \%$ registration tax on new vehicles. Panel (a) shows the baseline equilibrium prices of a new vehicles which are $\bar{P}=(183.12,380.25)$ (in thousands of DKK), with the implied before tax prices $(74.02,130.34)$. The secondary market adjusts to the high new car prices by raising the prices of used cars. Consumers respond by keeping their cars longer in aggregate, so cars are not scrapped until they are 13 and 21 years old. Panel (b) illustrates the equilibrium ownership distribution in the baseline. Poor consumers are affected by the high registration tax more: $8.32 \%$ of them do not own cars compared to $4.4 \%$ of rich consumers. These correspond to the observed shares of the outside good, and are calibrated together with the market shares of each car type. It easy to see from panel (b) that as expected, rich consumers sort into the higher quality car type and into newer cars of both types. The tax revenue in the baseline amounts to 18,016 DKK per household, or approximately 45.04 billion DKK in total. Its composition is shown in panel (c) of Figure 6. About $67.67 \%$ of all revenue comes from the sales tax, with more than two thirds of this part from the more expensive cars. 
Now consider the effect of reducing the registration tax rates by half. We use a calibrated version of the model to predict the impact of this change on equilibrium prices and scrappage ages, ownership distribution, driving, fuel consumption and $\mathrm{CO}_{2}$ emissions. Together with the decrease in the registration tax for new cars, in the counterfactual simulation we increase the fuel tax in order to keep the policy revenue-neutral. Taking all of the effects into account, we find that increasing the fuel tax by $12 \%$ is sufficient to compensate for the lost registration tax revenue.

The effects of the reform are presented in the bottom panels of Figure 6. There is a large reduction in the price of new vehicles visible in panel (a): after tax prices in the counterfactual economy are 137.82 and 271.59 thousand DKK which is a $24.7 \%$ and $28.6 \%$ reduction in price compared to the baseline case. The reduction in the cost of buying new cars has a significant effect on the longevity of car fleet: compared to the baseline, in the counterfactual economy the scrappage age falls by 2 years for the small car and by 4 years for the bigger car. Panel (e) shows that in the counterfactual simulation nearly all consumers own cars (the share of the outside good is $1.28 \%$ ), and there is a massive substitution away from the cheaper cars. This effect can be explained by the much higher utility provided by the type 2 cars. Even though these cars are significantly more expensive, they are also more durable, and the car-specific utility calibrated to match the baseline market shares is almost twice the level of the cheaper car. Evidently, in the baseline economy the high registration taxes made type 2 cars out of reach for a large fraction of the population, who substitute away from the cheaper car when the reduction in registration taxes cause new car prices to fall. Panel (f) presents the composition of tax revenue under the new policy. We can see that bigger cars becomes the dominant source of revenue, following the shift of ownership evident from panel (e). In addition, the share of fuel tax revenue increased from about $21 \%$ to $40 \%$, reflecting the higher total driving in the economy.

Table 1 provides more details on the effects of the tax rule changes. In particular, we can see that even though the average driving per person increases by about $16.4 \%$, the effect comes largely from the extensive margin. The estimated parameters do imply a moderate response to fuel prices with driving elasticities with respect to the fuel price in the range -0.1 to -0.3 (depending on consumer and car type). ${ }^{16}$ As a result of the relatively inelastic response in total

\footnotetext{
${ }^{16}$ Gillingham and Munk-Nielsen (2019) estimated a constant fuel price elasticity of -0.3 in their log specification which includes many additional controls and fixed effects. They show that the price elasticity is fairly robustly estimated across different specifications.
} 
Table 1: Effects on fuel consumption, driving and emissions

\begin{tabular}{|c|c|c|c|c|c|c|c|c|c|c|}
\hline & \multicolumn{2}{|c|}{$\begin{array}{c}\text { Market/ownership } \\
\text { shares }\end{array}$} & \multicolumn{2}{|c|}{$\begin{array}{c}\text { Driving } \\
\text { (1000 km/person) }\end{array}$} & \multicolumn{2}{|c|}{$\begin{array}{c}\text { Fuel consumption } \\
\text { (1000 liter/person) }\end{array}$} & \multicolumn{2}{|c|}{$\begin{array}{l}\mathrm{CO}_{2} \text { emissions } \\
\text { (ton/person) }\end{array}$} & \multicolumn{2}{|c|}{$\begin{array}{l}\text { Avg. elasticity } \\
\text { of driving }\end{array}$} \\
\hline & baseline & $\operatorname{sim}$ & baseline & $\operatorname{sim}$ & baseline & $\operatorname{sim}$ & baseline & $\operatorname{sim}$ & baseline & $\operatorname{sim}$ \\
\hline & \multicolumn{10}{|c|}{ Consumers with income above the median } \\
\hline Light cars (1) & 0.16 & 0.05 & 17.47 & 17.47 & 1.12 & 1.13 & 2.69 & 2.69 & -0.10 & -0.11 \\
\hline Heavy cars (2) & 0.30 & 0.45 & 19.86 & 19.90 & 1.47 & 1.48 & 3.52 & 3.53 & -0.10 & -0.11 \\
\hline \multirow[t]{2}{*}{ No car } & 0.04 & 0.00 & & & & & & & & \\
\hline & \multicolumn{10}{|c|}{ Consumers with income below the median } \\
\hline Light cars (1) & 0.18 & 0.06 & 15.78 & 15.70 & 1.02 & 1.01 & 2.43 & 2.42 & -0.25 & -0.27 \\
\hline Heavy cars (2) & 0.24 & 0.43 & 17.61 & 17.62 & 1.31 & 1.31 & 3.12 & 3.12 & -0.26 & -0.28 \\
\hline No car & 0.08 & 0.01 & & & & & & & & \\
\hline Total & 1.00 & 1.00 & 15.70 & 18.28 & 1.11 & 1.34 & 2.65 & 3.20 & & \\
\hline
\end{tabular}

Notes: The table shows the effects on the Danish automobile market from a reduction of the new car (registration) tax by half with simultaneous increase of the fuel tax rate by $12 \%$ to maintain the same amount of tax revenue before and after the policy change. The last column of the table shows the elasticity of driving with respect to fuel price, averaged over the holdings of the cars of different ages.

kilometers driven, the required increase in the fuel tax necessary for the tax change to be revenue neutral is comparatively moderate. However there are environmental costs to this policy change: the increase in driving results in higher total fuel consumption and $\mathrm{CO}_{2}$ emissions. ${ }^{17}$

To assess the welfare effects of the reform, we computed the expected value of the automobile fleet using $E V(a)$ values that follow from the solution of the consumer utility maximization problem, and the stationary ownership distribution $p(\bar{a}, P)$. The $50 \%$ reduction of the registration tax which is accompanied by a moderate $12 \%$ increase in price of fuel has a large positive effect on consumer welfare. In aggregate, the change in tax regime generates an annual welfare gain of approximately 9,302 DKK per capita or 23.3 billion DKK in total. Moreover, the welfare improvement is uniform across the types of consumers: the effect of the reform is equivalent to an annual lump sum payment of 4,606 DKK for the rich consumer, and 4,695 DKK for the poor consumer.

Of course, several caveats are in order from this stylized analysis, which was only crudely calibrated to approximate the Danish automobile market. First, the welfare gains mentioned above do not account for the substantial increase in $\mathrm{CO}_{2}$ emissions of 0.55 ton per person per year, corresponding to an $9 \%$ increase in total per capita $\mathrm{CO}_{2}$ emission in Denmark. If we were to consider a $\mathrm{CO}_{2}$-neutral policy change rather than a revenue neutral one, it would result in a much larger increase in fuel taxes. Second, there may be other effects from the tax change, such as increased road congestion, travel times, air pollution, and the effect on climate. We do

\footnotetext{
${ }^{17} \mathrm{CO}_{2}$ emissions are calculated based on the $2.392 \mathrm{~kg} / \mathrm{l}$ conversion rule.
} 
not take these into account. Our policy example should best be seen as a first cut illustration to demonstrate the potential usefulness of the model for policy analyses, and much deeper analysis would be required to reach any definitive conclusions on the desirability of this particular tax reform for Denmark.

\section{Equilibrium in the Primary Market for Automobiles}

We now extend our equilibrium framework to allow for endogenous determination of new vehicle prices. New car producers need to consider the effect of their pricing on both the new and used car markets and its effect on scrappage and equilibrium sorting of heterogeneous consumers into different types and ages of cars. We use this model to examine a stylized "merger to monopoly" counterfactual.

\subsection{Bertrand price competition between multi-product vehicle producers}

We make the common assumption of a Bertrand-Nash equilibrium, but allow new vehicle producers take into account the equilibrium in the secondary market including the endogenous determination of scrappage ages. Firms produce multiple vehicle types and internalize the effect of changing own car prices on not just competitor market shares, but also the composition of own sales.

As before, let $j \in\{1, \ldots, J\}$ index the set of all vehicles that could potentially be produced by $F$ firms which we index as $f \in\{1, \ldots, F\}$. Let the sets $\left(O_{1}, O_{2}, \ldots, O_{F}\right)$ be a partition of the set of possible vehicle types $\{1, \ldots, J\}$, so that $O_{f}$ denotes the set of vehicle types that could be produced by firm $f$. We can think of $O_{f}$ as a collection of production plans that firm $f$ owns, but whether it actually produces all $O_{f}$ vehicles is determined in equilibrium.

Let $c_{f}\left(\vec{q}_{f}\right)$ denote the cost function of be firm $f$, where $\vec{q}_{f}=\left\{q_{j}: j \in O_{f}\right\}$ is the vector of quantities of the vehicles that firm $f$ produces, normalized as shares of the population that buy these new vehicles in stationary equilibrium. We allow for very flexible patterns of production interdependencies, such as economies of scale and economies of scope due to central production of shared underlying vehicle components (e.g. engines, car bodies, etc). ${ }^{18}$ We take the set of vehicles $O_{f}$ (and their characteristics) as given, and assume there is common knowledge

\footnotetext{
${ }^{18}$ For simplicity, we ignore the higher level product R\&D investments that firms use to introduce new vehicle types into the market.
} 
by all automakers about how different types of consumers perceive different types of vehicles. That is, we assume that there is common knowledge of consumer preferences $u_{\tau}(j, a)$ and consumer marginal utilities of money, $\mu_{\tau}$ by all firms.

Let $\bar{P}_{f}=\left\{\bar{P}_{j}: j \in O_{f}\right\}$ be the vector of new vehicle prices that firm $f$ charges for its own vehicles types $j \in O_{f}$ in a stationary equilibrium, and let $\bar{P}_{-f}$ denote the new vehicle prices of firm $f$ competitors. In a Bertrand-Nash equilibrium, firm $f$ prices will be set as a best response $\bar{P}_{f}=\Psi_{f}\left(\bar{P}_{-f}\right)$ to the prices charged by its competitors:

$$
\begin{aligned}
\Psi_{f}\left(\bar{P}_{-f}\right)=\underset{\bar{P}_{f}}{\operatorname{argmax}}\left[\sum_{j \in O_{f}} \bar{P}_{j} q\left(\bar{a}_{j}\left(\bar{P}_{f}, \bar{P}_{-f}\right), j, \bar{P}_{f}, \bar{P}_{-f}\right)\right. & \\
& \left.-c_{f}\left(\left\{q\left(\bar{a}_{j}\left(\bar{P}_{f}, \bar{P}_{-f}\right), j, \bar{P}_{f}, \bar{P}_{-f}\right): j \in O_{f}\right\}\right)\right] .
\end{aligned}
$$

Equation (39) states that firm $f$ chooses the prices of its vehicles, $\bar{P}_{f}$, to maximize its profits, taking the prices of its competitors $\bar{P}_{-f}$ as given, while considering the effect of the prices on the overall equilibrium in the new and secondary market, including the possibility of cannibalization of sales of its own vehicles ("internal competition"). Though equation (39) provides only single period profits, in a stationary equilibrium if firm $f$ discounts future profits with discount factor $\beta_{f} \in(0,1)$, then discounted profits equal the right hand side of (39) scaled up by $1 /\left(1-\beta_{f}\right)$ which does not affect the definition of $\Psi_{f}$.

In equation (39), $q\left(\bar{a}_{j}\left(\bar{P}_{f}, \bar{P}_{-f}\right), j, \bar{P}_{f}, \bar{P}_{-f}\right)$ represents the new vehicle sales of type $j \in O_{f}$ in a stationary equilibrium of the model which is determined by the invariant ownership distribution $q(P)$ given in (22), see Theorem 2. The prices of all used vehicles will be implicit functions of $\left(\bar{P}_{f}, \bar{P}_{-f}\right)$ as described in Section 4. Thus, though each firm takes its competitor prices as given, each firm is presumed to have a sophisticated understanding of the equilibrium in the overall market, including prices, sales, scrappage ages, the stationary holdings distribution, and the effect of prices on the share of consumers who choose not to own a vehicle.

A Bertrand-Nash equilibrium is then any fixed point $\left(\bar{P}_{1}^{*}, \ldots, \bar{P}_{F}^{*}\right)$ to the system of best response correspondences for the $F$ firms in this market:

$$
\left(\bar{P}_{1}^{*}, \ldots, \bar{P}_{F}^{*}\right) \in\left(\Psi_{1}\left(\bar{P}_{-1}^{*}\right), \ldots, \Psi_{F}\left(\bar{P}_{-F}^{*}\right)\right)
$$


Figure 7: Bertrand-Nash Equilibrium in the new car market

(a) Demands as function type 1 car price

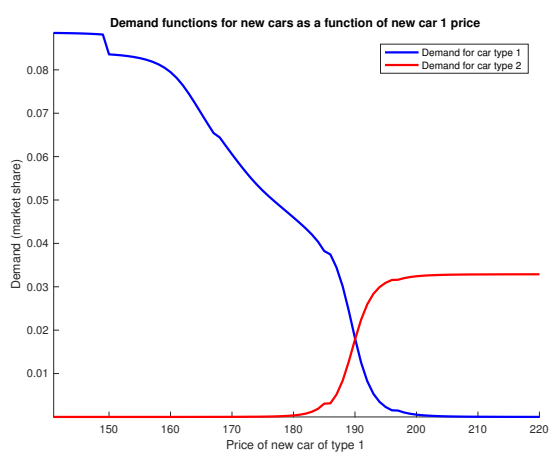

(c) Scrappage age of type 1 car

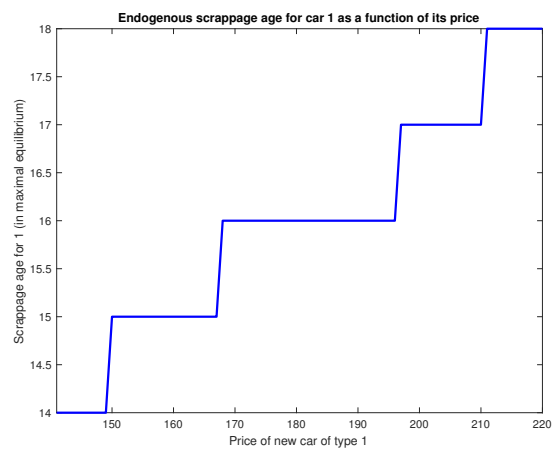

(b) Market share of outside good

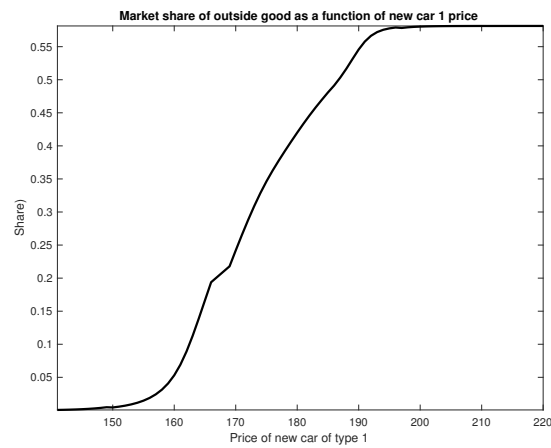

(d) Best response functions

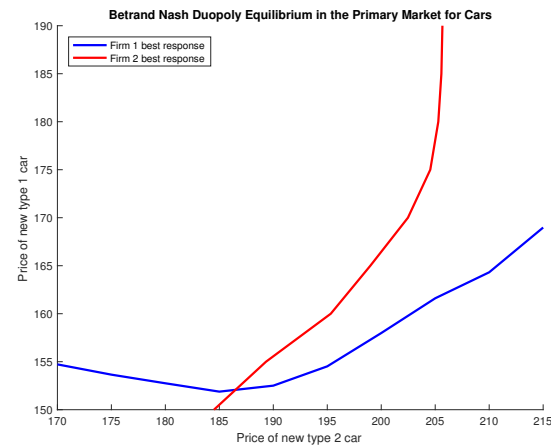

\subsection{Illustration: analysis of new car duopoly and impact of merger to monopoly}

Consider a market with $J=2$ types of vehicles and $F=2$ firms and assume the market is initially a Bertrand-Nash duopoly. Panels (a) to (c) of Figure 7 show how the Bertrand-Nash equilibrium depends on the price of new car of type $1, \bar{P}_{1}$. Panel (a) plots the demand for both car types when firm 1 changes the price of its vehicle $\bar{P}_{1}$, while firm 2 keeps its vehicle price fixed at $\bar{P}_{2}=220$. We see, as expected, that new car 1 demand is a downward sloping function of its own price, whereas the demand for new cars of type 2 is an increasing function of the price of type 1 , reflecting a market-level substitution effect. Notice that demand for vehicle type 2 only arises when $\bar{P}_{1} \geq 180$. When $\bar{P}_{1}$ rises to 220 (and thus equal to $\bar{P}_{2}$ ), demand for vehicle type 1 falls to zero, and the demand for vehicle type 2 peaks at a little over $3 \%$ of the population.

Panels (b) and (c) of Figure 7 provide insight into the complexity of demand when all relevant avenues of consumer substitution are considered. It follow from panel (c) that when 
Table 2: Welfare analysis of the hypothetical merger to monopoly

\begin{tabular}{|c|c|c|c|c|}
\hline & \multicolumn{2}{|c|}{ Monopoly } & \multicolumn{2}{|c|}{ Duopoly } \\
\hline & car type 1 & car type 2 & car type 1 & car type 2 \\
\hline Price of new car & 374.91 & 386.93 & 151.84 & 186.27 \\
\hline Fraction of new cars, $\%$ & $1.5 \times 10^{-4}$ & 1.5 & 0.9 & 6.7 \\
\hline \multirow[t]{2}{*}{ Scrappage age } & 18 & 20 & 15 & 18 \\
\hline & \multicolumn{4}{|c|}{ Rich consumers } \\
\hline Fraction owning no car, \% & \multicolumn{2}{|c|}{2.4} & \multicolumn{2}{|c|}{0.0} \\
\hline Distribution of cars held, $\%$ & $7 \times 10^{-3}$ & 97.6 & 11.5 & 88.5 \\
\hline Average age held & 7.80 & 7.85 & 2.00 & 2.15 \\
\hline \multirow[t]{2}{*}{ Consumer surplus } & 0.002 & 32.54 & 2.66 & 26.33 \\
\hline & \multicolumn{4}{|c|}{ Poor consumers } \\
\hline Fraction owning no car, \% & \multicolumn{2}{|c|}{96.8} & \multicolumn{2}{|c|}{0.04} \\
\hline Distribution of cars held, $\%$ & 0 & 3.2 & 10.5 & 89.1 \\
\hline Average age held & 17.15 & 18.27 & 8.8 & 9.9 \\
\hline Consumer surplus & 0.0004 & 6.69 & 3.23 & 42.26 \\
\hline Profits & \multicolumn{2}{|c|}{3.35} & \multicolumn{2}{|c|}{1.11} \\
\hline Consumer surplus & \multicolumn{2}{|c|}{39.24} & \multicolumn{2}{|c|}{74.48} \\
\hline Total social surplus & \multicolumn{2}{|c|}{42.59} & \multicolumn{2}{|c|}{75.59} \\
\hline
\end{tabular}

$\bar{P}_{1}$ rises, the secondary market adjusts and the scrappage age $\bar{a}_{1}\left(\bar{P}_{1}, \bar{P}_{2}\right)$ increases from 14 to 18. The increase in the scrappage age reduces new vehicle demand and contributes to the price elasticity of demand for new vehicles. Panel (b) shows an additional margin of substitution: an increasing share of consumers choosing not to own vehicles as $\bar{P}_{1}$ rises from nearly zero to about $60 \%$ when $\bar{P}_{1}$ approaches the price of vehicle $2, \bar{P}_{2}=220$. Essentially all of these are in the $80 \%$ of the population who are poor, i.e. have the higher marginal utility of money $\mu_{\tau}=2$ compared to $\mu_{\tau}=1$ for the rich consumers.

Panel (d) of Figure 7 illustrates the existence of a unique duopoly Bertrand-Nash equilibrium in this model by plotting the two best response functions $\Psi_{1}$ and $\Psi_{2}$ as a function of the competitor's price. We assume that the two firms have constant marginal costs of production given by $c_{1}=150$ for vehicle type 1 and $c_{2}=170$ for vehicle type 2 . The reaction functions cross at the pair $\left(\bar{P}_{1}^{*}, \bar{P}_{2}^{*}\right)=(151.84,186.27)$. In this equilibrium, vehicle type 1 is actually the "inferior good" in the sense that even though it has a lower marginal cost of production and a lower price, both consumer types prefer vehicle type 2 at the higher price of $\bar{P}_{2}^{*}=186.27$. The markup for vehicle type 1 is only 1.84 whereas the markup for vehicle type 2 is 16.27 . The higher utility provided by vehicle type 2 makes it relatively more valuable to consumers despite its higher price.

Table 2 summarizes key statistics from a "merger to monopoly" counterfactual where firms 1 and 2 merge. The table shows that allowing such a merger would have devastating impact 
on the car market: the prices of cars 1 and 2 more than double. The monopolist endogenously "overprices" vehicle type 1, causing its market share to fall nearly to zero. In response to the high prices charged by the monopolist, nearly $78 \%$ of the population chooses to forgo owning a car after the merger. Though firm profits triple after the merger, consumer surplus is nearly cut in half and total social surplus (consumer surplus plus total profits) falls by $44 \%$. Since consumer surplus is vastly greater than firm profits, it seems that allowing a merger would be a very bad idea in this stylized economy.

\section{Discussion and Conclusions}

We have introduced a computationally tractable model of equilibrium in the primary and secondary markets for automobiles that allows for flexible specifications of preferences and consumer heterogeneity and transactions costs. Our work was inspired by the early static discrete choice models of equilibrium in the automobile market pioneered by Manski, Sherman, and Berkovec and the subsequent efforts to extend their models to include dynamics and transactions costs and model equilibrium price setting in the primary market by Rust, Stolyarov, Gavazza, Lizzeri and Roketskiy, and Esteban and Shum. We believe that our framework is promising for empirical applications and policy analysis, and in future work we plan to further extend and apply it in a number of directions.

One of these directions is ongoing work (Gillingham, Iskhakov, Munk-Nielsen, Rust and Schjerning, 2019) to structurally estimate an "overlapping generations" version of our model using Danish register data to allow for a realistic counterfactual analysis of vehicle tax reform in Denmark. Another direction is to develop alternatives to the Berry, Levinsohn and Pakes (1995) approach for estimating consumer preferences and analyzing equilibrium, by analyzing the identification of parameters in the context of a realistic dynamic model of the primary and secondary automobile markets. A third direction would relax the assumption of stationarity and extend out concept of equilibrium to allow for macroeconomic shocks that can capture the pronounced "waves" often found in the age distribution of vehicles (Adda and Cooper, 2000). We are comparing different solution concepts in terms of computational tractability and empirical realism, including the "temporary equilibrium" concept of Grandmont (1977), the "sufficient statistic" approach of Krusell and Smith (1998), as well as a full blown rational expectations equilibrium that takes into account the entire holdings distribution of cars as a 
component of the "state variables" that consumers use to predict future prices as in Cao (2016).

A very challenging extension of our model would endogenize the characteristics of vehicles by allowing firms to invest in R\&D to produce new vehicle designs. Longer-run competition on attributes will likely require a fundamentally non-stationary framework and raises questions of consumer expectations over future products. Very promising headway into this sort of analysis has been done in the pioneering work of Goettler and Gordon (2011), and it may be possible to adapt this approach into a more evolutionary model of the automobile market. A final challenging extension would be to incorporate asymmetric information in a more detailed treatment of the "microstructure" of trade in the automobile market, including endogenous intermediation of trade by car dealers as well as direct consumer transactions. Recent studies such as Biglaiser, Li, Murry and Zhou (2019) have provided new empirical insights into the microstructure of trade that are not modeled in our framework, but represent important directions to pursue in the development of more detailed and realistic models of trade in automobile markets.

\section{References}

AdDA, J. And R. CoOper (2000): "Balladurette and Juppette: A Discrete Analysis of Scrapping Subsidies," Journal of Political Economy, 108(4), 778-806.

AKerlof, G. A. (1970): “The Market for 'Lemons': Quality Uncertainty and the Market Mechanism," Quarterly Journal of Economics, 84, 488-500.

Berkovec, J. (1985): "New Car Sales and Used Car Stocks: A Model of the Automobile Market," RAND Journal of Economics, 16(2), 195-214.

Berry, S., J. Levinsohn and A. Pakes (1995): “Automobile Prices in Market Equilibrium," Econometrica, 63(4), 841-890.

Biglaiser, G., F. Li, C. Murry and Y. Zhou (2019): "Middlemen as Information Intermediaries: Evidence from Used Car Markets," manuscript, Stony Brook University.

CAO, D. (2016): “Recursive Equilibrium in Krusell and Smith (1998),” manuscript.

Esteban, S. and M. Shum (2007): "Durable-goods Oligopoly with Secondary Markets: The Case of Automobiles," RAND Journal of Economics, 38(2), 332-354. 
GavazZA, A., A. LizZeri and N. Roketskiy (2014): “A Quantitative Analysis of the UsedCar Market," American Economic Review, 104(11), 3668-3700.

Gillingham, K., F. Iskhakov, A. Munk-Nielsen, J. Rust and B. Schjerning (2019): “A Dynamic Model of Vehicle Ownership, Type Choice, and Usage," manuscript.

Gillingham, K. and A. MunK-Nielsen (2019): "A tale of two tails: Commuting and the fuel price response in driving," Journal of Urban Economics, 109, 27-40.

Goettler, R. L. And B. R. Gordon (2011): “Does AMD Spur Intel to Innovate More?," Journal of Political Economy, 119(6), 1141-1200.

Grandmont, J. M. (1977): “Temporary General Equilbrium Theory,” Econometrica, 45(3), $535-572$.

Hendel, I. And A. LiZzeri (1999): “Adverse Selection in Durable Goods Markets,” American Economic Review, 89(5), 1097-1115.

Howard, R. A. (1960): Dynamic Programming and Markov Processes. MIT Press, Cambridge, MA.

IskHAkov, F., J. Rust and B. SchJERning (2015): "Recursive Lexicographical Search: Finding All Markov Perfect Equilibria of Finite State Directional Dynamic Games," The Review of Economic Studies, 83(2), 658-703.

KONISHI, H. And M. SANDFORT (2002): "Existence of Stationary Equilibrium in the Markets for New and Used Durable Goods," Journal of Economic Dynamics and Control, 26(6), 1029-1052.

Krusell, P. And T. SMith (1998): "Income and Wealth Heterogeneity in the Macroeconomy," Journal of Political Economy, 106(5), 867-896.

MAnsKi, C. (1980): "Short Run Equilibrium in the Automobile Market," Falk Institute Discussion Paper 8018, Hebrew University of Jerusalem.

(1983): "Analysis of Equilibrium Automobile Holdings in Israel with Aggregate Discrete Choice Models," Transportation Research, 17B (5), 373-389.

MAnski, C. And E. Goldin (1983): “An Econometric Analysis of Vehicle Scrappage,” Transportation Science, 17 (4), 365-375. 
MAnSKI, C. And L. Sherman (1980): "Forecasting Equilibrium Motor Vehicle Holdings by Means of Disaggregate Models," Transportation Research Record, 764, 96-103.

MCFADDEN, D. (1981): “Econometric Models of Probabilistic Choice," in Structural Analysis of Discrete Data, ed. by C. Manski and D. McFadden, pp. 198-272. MIT Press, Cambridge, Massachusetts.

RUST, J. (1985a): “Equilibrium holdings distributions in durable asset markets," Transportation Research B, 19(4), 331-345.

(1985b): "Stationary Equilibrium in a Market for Durable Assets," Econometrica, 53(4), 783-806.

(1985c): "When is it Optimal to Kill Off the Market for Used Durable Goods?," Econometrica, 54(1), 65-86.

(1987): “Optimal Replacement of GMC Bus Engines: An Empirical Model of Harold Zurcher," Econometrica, 55(5), 999-1033.

(1994): "Structural estimation of markov decision processes," vol. 4 of Handbook of Econometrics, pp. 3081 - 3143. Elsevier.

Stolyarov, D. (2002): “Turnover of Used Durables in a Stationary Equilibrium: Are Older Goods Traded More?," Journal of Political Economy, 110(6), 1390-1413. 


\section{Appendix}

\section{A Homogeneous Consumer Economy (replication of Rust 1985b)}

In this appendix we replicate the results from Rust (1985b) for our case of discrete product market. This is a limiting case for the general framework that we incrementally build in the main part of the paper which admits a simple numerical solution that constitutes the source of precise starting values for the main solution algorithm.

\section{Model setup}

Consider the model set up in Section 3, with the simplification that (i) transaction costs are zero, (ii) there is no outside option, and most importantly (iii) there are no extreme value preference shocks.

Denote $V(a)$ the value function for the owner of car of age $a \in\{1, \ldots, A-1\}$. Consumer's decision making process is given by the following Bellman equation:

$$
\begin{aligned}
V(a) & =\max \left\{v(a, \kappa) ; \max _{d \in\left\{0, \ldots, \gamma^{\star}-1\right\}} v(a, d)\right\}, \\
v(a, \kappa) & =u(a)+\beta(1-\alpha(a)) V(a+1)+\beta \alpha(a) V(A), \\
v(a, d) & =u(d)-\mu[P(d)-P(a)]+\beta(1-\alpha(d)) V(d+1)+\beta \alpha(d) V(A),
\end{aligned}
$$

where $v(a, \cdot)$ is the choice specific value function, and $d=\kappa$ denotes the choice of keeping the existing car. For the owners of the maximum age car, $a=A$, the keep choice is not available, and thus the first component in the right hand side of (41) disappears.

Note the timing convention underlying the Bellman equation given by (41)-(43). We assume that the consumer enter the period with a car of age $a$, and makes the trading/keeping choice immediately at the start of the period. The car that consumer ends up with after trading is the one that is utilized in the period, and that can be involved in the accident during that time. By the start of the next period this car is one year older, or in case of an accident reaches the upper bound of age $A$ and can not be kept any longer (must be scrapped). With this timing convention, it is impossible to start the period with a brand new car, thus the state variable $a$ can only take values from $\{1, \ldots, A\}$. In the same time, cars that can be acquired during trading can have ages $\left\{0, \ldots, \gamma^{\star}-1\right\}$ which includes the new cars of age zero.

Theorem A.T1 (Consumer choice in absence of transaction cost). Suppose the price of the new car is given by $P(0)=\bar{P}$ and $P(a)=\underline{P}$ for $a \geq \gamma^{\star}$ for some $\gamma^{\star} \leq A$. With no transaction costs, the optimal policy $a^{\star}(a)$ in the car replacement problem (41)-(43) for $a \in\left\{1, \ldots, \gamma^{\star}-1, A\right\}$ does not depend on a, i.e. $a^{\star}(a)=a^{\star}$, and provided that $a^{\star} \in\left\{0, \ldots, \gamma^{\star}-2\right\}$, it holds:

$$
\begin{aligned}
V\left(a^{\star}\right) & =\frac{u\left(a^{\star}\right)}{1-\beta}-\frac{\mu \beta}{1-\beta}\left[P\left(a^{\star}\right)-\left(1-\alpha\left(a^{\star}\right)\right) P\left(a^{\star}+1\right)-\alpha\left(a^{\star}\right) \underline{P}\right], \\
V(a) & =V\left(a^{\star}\right)-\mu\left[P\left(a^{\star}\right)-P(a)\right] .
\end{aligned}
$$

In the special case of $a^{\star}=\gamma^{\star}-1$ an additional condition applies: if for $V\left(a^{\star}\right)=V\left(\gamma^{\star}-1\right)$ defined by (44) it holds $V\left(a^{\star}\right)-V\left(\gamma^{\star}\right)<\mu\left[P\left(\gamma^{\star}-1\right)-\underline{P}\right], V\left(\gamma^{\star}-1\right)$ is instead given by:

$$
V\left(\gamma^{\star}-1\right)=\frac{u\left(\gamma^{\star}-1\right)}{1-\alpha\left(\gamma^{\star}-1\right) \beta}+\beta \frac{1-\alpha\left(\gamma^{\star}-1\right)}{1-\alpha\left(\gamma^{\star}-1\right) \beta} V\left(\gamma^{\star}\right)-\frac{\mu \alpha\left(\gamma^{\star}-1\right) \beta}{1-\alpha\left(\gamma^{\star}-1\right) \beta}\left[P\left(\gamma^{\star}-1\right)-\underline{P}\right] .
$$


Proof. First, note that for $a \in\left\{1, \ldots, \gamma^{\star}-1\right\}$ the value of keeping represented by the first component in the outer maximization operator in (41) is in fact included into the inner maximization in the second component. This does not hold for $a \in\left\{\gamma^{\star}, \ldots, A-1\right\}$ because the cars of these ages are not traded on the market. Yet, the same does hold for $A$ because the option to keep is simply not available for the cars of maximum age.

To shorted notation, define $E V(d)=(1-\alpha(d)) V(d+1)+\alpha(d) V(A)$.

Consider cars of ages $a \in\left\{1, \ldots, \gamma^{\star}-1, A\right\}$. Because the choice set in (41) is finite, there exist an optimal replacement car $a^{\star}(a)$ for every existing car. Suppose that for some $a \neq a^{\prime}$ we have $a^{\star}(a) \neq a^{\star}\left(a^{\prime}\right)$. From the definition of $a^{\star}(a)$ applied at point $a$ it follows:

$u\left(a^{\star}(a)\right)-\mu\left[P\left(a^{\star}(a)\right)-P(a)\right]+\beta E V\left(a^{\star}(a)\right) \geq u\left(a^{\star}\left(a^{\prime}\right)\right)-\mu\left[P\left(a^{\star}\left(a^{\prime}\right)\right)-P(a)\right]+\beta E V\left(a^{\star}\left(a^{\prime}\right)\right)$.

In the same time from the definition of $a^{\star}\left(a^{\prime}\right)$ applied at point $a^{\prime}$ it follows:

$u\left(a^{\star}\left(a^{\prime}\right)\right)-\mu\left[P\left(a^{\star}\left(a^{\prime}\right)\right)-P\left(a^{\prime}\right)\right]+\beta E V\left(a^{\star}\left(a^{\prime}\right)\right) \geq u\left(a^{\star}(a)\right)-\mu\left[P\left(a^{\star}(a)\right)-P\left(a^{\prime}\right)\right]+\beta E V\left(a^{\star}(a)\right)$.

Because both $P(a)$ and $P\left(a^{\prime}\right)$ cancel out in the inequalities above, we have a contradiction. Thus, $a^{\star}(a)=a^{\star}$ is independent of $a$.

Next, consider $a \in\left\{1, \ldots, \gamma^{\star}-1\right\}, a^{\star} \in\left\{1, \ldots, \gamma^{\star}-2\right\}$. From (41) and (43) we have

$$
\begin{aligned}
V\left(a^{\star}\right) & =u\left(a^{\star}\right)+\beta E V\left(a^{\star}\right)=u\left(a^{\star}\right)+\beta\left(1-\alpha\left(a^{\star}\right)\right) V\left(a^{\star}+1\right)+\beta \alpha\left(a^{\star}\right) V(A), \\
V(a) & =u\left(a^{\star}\right)-\mu\left[P\left(a^{\star}\right)-P(a)\right]+\beta E V\left(a^{\star}\right) \\
& =V\left(a^{\star}\right)-\mu\left[P\left(a^{\star}\right)-P(a)\right], \forall a \in\left\{1, \ldots, \gamma^{\star}-1, A\right\}, \\
V\left(a^{\star}+1\right) & =u\left(a^{\star}\right)-\mu\left[P\left(a^{\star}\right)-P\left(a^{\star}+1\right)\right]+\beta E V\left(a^{\star}\right), \\
V(A) & =u\left(a^{\star}\right)-\mu\left[P\left(a^{\star}\right)-\underline{P}\right]+\beta E V\left(a^{\star}\right), \text { and then combining with the first line } \\
E V\left(a^{\star}\right) & =\frac{1}{1-\beta}\left(u\left(a^{\star}\right)-\mu\left[P\left(a^{\star}\right)-\left(1-\alpha\left(a^{\star}\right)\right) P\left(a^{\star}+1\right)-\alpha\left(a^{\star}\right) \underline{P}\right]\right) .
\end{aligned}
$$

Plugging the last line into the first gives (44).

The case of $a^{\star}=\gamma^{\star}-1$ is special because we have not excluded the possibility for the choice to keep to be optimal for $a^{\star}+1=\gamma^{\star}$, and so the expression for $V\left(a^{\star}+1\right)$ above does not hold. In this case the maximum in (41) is attained at the first component and thus it holds $V\left(\gamma^{\star}\right)>u\left(\gamma^{\star}-1\right)+\beta E V\left(\gamma^{\star}-1\right)-\mu\left[P\left(a^{\star}\right)-\underline{P}\right]$, where $E V\left(a^{\star}\right)=E V\left(\gamma^{\star}-1\right)$ is defined in the last equation above. Formula (46) is then obtain by substituting $V(A)$ and simplifying the expression for $V\left(a^{\star}\right)$ :

$$
V\left(\gamma^{\star}-1\right)=u\left(\gamma^{\star}-1\right)+\beta\left(1-\alpha\left(\gamma^{\star}-1\right)\right) V\left(\gamma^{\star}\right)+\beta \alpha\left(\gamma^{\star}-1\right) V(A) .
$$

This completes the proof of Theorem A.T1.

According to equation (44) the value of owning (and perpetual switching back to) the optimal car $a^{\star}$ is given by the infinite discounted stream of utilities $u\left(a^{\star}\right)$ plus the infinite discounted stream of monetary costs to replace, which is paid from the next period (thus additional $\beta$ ) and take into account the possibility of accident. The maximum utility of the owner of an arbitrary car $a$ given by (45) equals the monetary cost of replacing the existent car with the optimal one, and following the strategy of perpetual switching back to it.

Even though Theorem A.T1 does not go as far as providing the exact formula for the optimal replacement policy for the problem (41)-(43) as a function of model fundamentals 
$\{u(\cdot), \beta, \alpha(\cdot), P(\cdot)\}$, it provides all the necessary components. Plugging (44) and (45) into (43) to compute choice specific values $v(a, d)$ for $d \in\left\{0, \ldots, \gamma^{\star}-1\right\}$ is sufficient to calculate the optimal replacement choice. The first statement of Theorem A.T1 ensures that doing this calculation once for $a=1$ is sufficient. The special case arises when the optimal car is just one year before the scrap age, and its market price is sufficiently higher than the scrap value $\underline{P}$ : to the extent that it becomes optimal to keep it for at least one extra year. This is equivalent to $V\left(\gamma^{\star}\right)$ given by (41) to attain maximum at the first component of the maximum operator. The value function $V\left(\gamma^{\star}\right)$ can be found numerically in $A-\gamma^{\star}+1$ steps of backward recursion over car age $a$, starting from $V(A)$ which is given by (45). ${ }^{19}$

Note that outside of the special case, Theorem A.T1 is silent about the solution at points $a \in\left\{\gamma^{\star}, \ldots, A-1\right\}$. However, as it will be clear below, there results are sufficient to analytically characterize the equilibrium in the economy with homogeneous consumers.

Definition A.D1. A stationary equilibrium is given by the triplet $\left\{q\left(\cdot, \zeta^{\star}\right), P(\cdot), \zeta^{\star}\right\}$ where $q \in$ $\mathbb{R}^{A}$ and $P \in \mathbb{R}^{A+1}$ are vectors, and $\zeta^{\star} \in\{1, \ldots, A-1\}$ is a constant, which satisfy:

1. The new car price is given by $P(0)=\bar{P}$;

2. The scrap value is given by $\underline{P}=P(a)$ for all $a \geq \zeta^{\star}$;

3. $q\left(\zeta^{\star}\right)$ is a stationary holdings distribution which constitutes a fixed point of the linear operator defined in (A) by the physical transition matrix $Q$, so it holds $q\left(\zeta^{\star}\right)=q\left(\zeta^{\star}\right) Q$;

4. Each consumer follows a replacement policy $d^{\star}(a):\{1, \ldots, A\} \rightarrow\{\kappa, 0, \ldots, A-1\}$, such that for all $a$ it returns an element of the set of maximizers of the Bellman equation (41), so it holds $\forall a \in\{1, \ldots, A\} d^{\star}(a) \in \operatorname{argmax}_{d \in\{\kappa, 0, \ldots, A-1\}} v(a, d)$, where $v(a, d)$ is defined in (42)-(43);

5. The policy functions $d^{\star}(a)$ satisfies the market clearing condition:

$$
\sum_{a=1}^{A} q\left(\zeta^{\star}, a\right) \mathbb{1}\left\{d^{\star}(a)=a^{\prime}\right\}=1-\mathbb{1}\left\{d^{\star}\left(a^{\prime}\right)=\kappa\right\}, \forall a^{\prime} \in\{1, \ldots, A-1\} .
$$

\section{Welfare maximizing car replacement}

Before proceeding to the characterization of the equilibrium we have to consider problem (41) in the absence of used car market. In this specification consumers are not able to trade on the secondary market, and so in every period they only have to decide whether to keep their old car of age $a$ or to replace it with a brand new one. Solution to this problem will have a major role for determining the equilibrium in the homogeneous consumer economy.

As before the price of the new car is $P(0)=\bar{P}$, and set the value of a car of age $a>0$ to be $P(a)=\underline{P}$. The Bellman equation for $a \in\{0, \ldots, A-1\}$ is given by

$$
\begin{aligned}
& W(a)=\max \{u(a)+\beta(1-\alpha(a)) W(a+1)+\beta \alpha(a) W(A) \\
& u(0)-\mu(\bar{P}-\underline{P})+\beta(1-\alpha(0)) W(1)+\beta \alpha(0) W(A)\},
\end{aligned}
$$

where the first component under the maximization operator corresponds to the decision to keep $(d=\kappa)$, and the second to replace $(d=0)$. At the terminal age cars can only be replaced, thus $W(A)=u(0)-\mu(\bar{P}-\underline{P})+\beta W(1)$.

\footnotetext{
${ }^{19}$ In fact, all value functions $V(a), a \in\left\{\gamma^{\star}, \ldots, A-1\right\}$ will be computed by the described procedure which is an application of the state recursion algorithm (Iskhakov, Rust and Schjerning, 2015).
} 
In the economy with identical consumers which therefore does not generate any gains from trade, the value function $W(a)$ captures the maximum attainable welfare of the consumer owning car of age $a$. Thus, the problem (48) can be interpreted as the problem faced by a social planner who must choose optimal replacement policy to maximize consumer welfare.

Denote $W(0)$ the value stemming from the endowment of a new car in period zero. Evaluating (48) at $a=0$ we have $W(0)=u(0)+\beta(1-\alpha(0)) W(1)+\beta \alpha(0) W(A)$, corresponding to the decision to keep. With this, the second component in (48) can be interpreted as the value of new car net of trade cost, which is independent of $a$. Then, given our assumption of a nonincreasing utility function, i.e. $u(a+1) \leq u(a)$ for $a \in 0, \ldots, A$, it is easy to show by backward induction on car age that $W(a)$ is a non-increasing function of $a$. This ensures the existence of car age $\delta^{\star}$ such that $W\left(\delta^{\star}-1\right)$ in (48) is equal to the first component, and $W\left(\delta^{\star}\right)$ to the second component under maximum. In other words, $\delta^{\star}$ is the smallest age of a car that is replaced under the optimal policy, i.e.

$$
\delta^{\star}=\min \{a \in\{1, \ldots, A\}: W(a)=W(0)-\mu(\bar{P}-\underline{P})\} .
$$

The socially optimal replacement policy is then given by "keep" when $a<\delta^{\star}$ and "replace" when $a \geq \delta^{\star}$. The optimal threshold $\delta^{\star}$ can be calculated numerically using (49) once the value function $W(a)$ is computed. Standard dynamic programming technique applies: the Bellman operator defined by (48) is a contraction mapping, and so $W(a)$ is a unique fixed point of this operator. As it will be clear in the next section, both object are crucial for market equilibrium.

\section{Characterization of equilibrium in the homogeneous consumer economy}

Theorem A.T2 (Equilibrium in homogeneous consumer economy). The unique stationary equilibrium $\left\{q\left(\gamma^{\star}\right), P(a), \gamma^{\star}\right\}$ in the economy with homogeneous consumers whose decision process is given by (41)-(43), is composed of:

1. Scrappage age $\gamma^{\star}$ equal to the optimal replacement age in problem (48), as defined in (49), so it holds $\gamma^{\star}=\delta^{\star}$;

2. Non-increasing price function $P(a)$ defined by

$$
P(a)= \begin{cases}\bar{P}-\frac{1}{\mu}(W(0)-W(a)), & a \in\left\{1, \ldots, \gamma^{\star}-1\right\} \\ \underline{P}, & a \in\left\{\gamma^{\star}, \ldots, A\right\}\end{cases}
$$

where $W(a)$ is the unique fixed point of the Bellman operator (48);

3. Holding distribution $q\left(\gamma^{\star}\right)$ which is unique invariant distribution corresponding to the transition matrix $Q\left(\gamma^{\star}\right)$, so it holds $q\left(\gamma^{\star}\right)=q\left(\gamma^{\star}\right) Q\left(\gamma^{\star}\right)$. $Q\left(\gamma^{\star}\right)$ is given by (A), but with row $i=\gamma^{\star}$ replaced with vector $\left(1-\alpha\left(\gamma^{\star}\right), 0, \ldots, 0, \alpha\left(\gamma^{\star}\right)\right)$.

Proof. Let the scrappage age $\gamma^{\star}$ equal to the optimal replacement age $\delta^{\star}$ defined in (49).

Following the definition of the stationary equilibrium A.D1 (page 50), first observe that from (50) in follows $P(0)=\bar{P}$ and, due to (49), $P\left(\gamma^{\star}\right)=\underline{P}$. Thus, the first two conditions of A.D1 are satisfied.

Because the optimal choice $a^{\star}$ is independent of existing car age $a$ (by Theorem A.T1), any stationary age distribution when $A>1$, and cars are changing age, requires that consumers are indifferent between all replacement options. Therefore, next we show that price function $P(a)$ defined by (50) guarantees this property, which also ensures that conditions 4 and 5 in A.D1 are satisfied. 
Recall that for $a \in\left\{1, \ldots, \gamma^{\star}-1\right\}$ the decision to keep is optimal, thus:

$$
W(a)=u(a)+\beta(1-\alpha(a)) W(a+1)+\beta \alpha(a) W(A) .
$$

Subtracting this equation component-wise from $W(0)=(1-\beta) W(0)+\beta(1-\alpha(a)) W(0)+$ $\beta \alpha(a) W(0)$, and combining with the definition of price function (50) we get

$$
\begin{aligned}
\mu[\bar{P}-P(a)] & =(1-\beta) W(0)-u(a)+\beta(1-\alpha(a)) \mu[\bar{P}-P(a+1)]+\beta \alpha(a) \mu[\bar{P}-\underline{P}] \\
P(a) & =\frac{u(a)-(1-\beta) W(0)}{\mu}+(1-\beta) \bar{P}+\beta(1-\alpha(a)) P(a+1)+\beta \alpha(a) \underline{P} .
\end{aligned}
$$

Plugging the last equation into (45) and using (50) again, we have

$$
\begin{aligned}
V(a)=\frac{u\left(a^{\star}\right)}{1-\beta}-\frac{\mu}{1-\beta}\left[\frac{u\left(a^{\star}\right)-(1-\beta) W(0)}{\mu}+(1-\beta) \bar{P}\right]+\mu P(a) & = \\
=W(0)-\mu P & +\mu P(a)=W(a) .
\end{aligned}
$$

From (43) it follows then for $a \in\left\{1, \ldots, \gamma^{\star}-1\right\}$ and $d \in\left\{0, \ldots, \gamma^{\star}-2\right\}$ :

$$
v(a, d)=u(d)-W(d)+W(a)+\beta(1-\alpha(d)) W(d+1)+\beta \alpha(d) W(A)=W(a),
$$

where we again use (51) and the definition of price function (50). Equations (52) and (53) together show that the maximum expected discounted utility $V(a)=W(a)$ of the owner of car of age $a$ is attained by choosing any $d \in 0, \ldots, \gamma^{\star}-2$. Because in the terms of Theorem A.T1 $a^{\star}<\gamma^{\star}-1$, the special case does not apply. Therefore equation (53) holds for $a=\gamma^{\star}$, and $d=\gamma^{\star}-1$ as well. It is also straightforward to verify that $V\left(\gamma^{\star}\right)=W\left(\gamma^{\star}\right)$.

We have thus shown that in the considered equilibrium the consumers who own a car of age $a \in\left\{1, \ldots, \gamma^{\star}\right\}$ are indifferent between replacing to any car of age $d \in\left\{0, \ldots, \gamma^{\star}-1\right\}$, and the maximum attainable utility is given by $V(a)=W(a)$.

The non-increasing property of the price function (50) follows directly from the non-increasing property of $W(a)$. Given our assumption of a non-increasing utility function, $u(a+1) \leq u(a)$ for $a \in 0, \ldots, A$, this can in turn be established by mathematical induction starting from $W(A)=$ $\cdots=W\left(\gamma^{\star}\right)=W(0)-\mu[\bar{p}-\underline{P}]$ and going backwards in $a$.

The stationary holdings distribution follows from direct application of the condition 3 in A.D1. The definition of equilibrium physical transition matrix $Q\left(\gamma^{\star}\right)$ reflects the fact that no cars are kept past scrappage age $\gamma^{\star}$ under the optimal replacement strategy described above. When $\alpha(a)=0$ for all ages, the unique invariant distribution $q\left(\gamma^{\star}\right)$ is uniform with the unit mass distributed equally between points $a \in\left\{1, \ldots, \gamma^{\star}\right\}$. With positive probability of accidents, invariant distribution is unique under assumption of irreducibility of the Markov chain implied by the physical transition matrix $Q$, which is satisfied when $0 \leq \alpha(a)<1$ for every $a$.

The uniqueness of the stationary equilibrium in the homogeneous consumer economy of automobiles follows from the uniqueness of the invariant fleet age distribution.

Corollary A.C1. In the equilibrium in the homogeneous consumer economy with no transaction costs defined in Theorem A.T2, consumers are indifferent between replacing their existing car with the car of any age available in the economy. This holds for owners of all ages of cars with positive shares in the stationary fleet age distribution.

Corollary A.C2. The equilibrium in the homogeneous consumer economy with no transaction costs defined in Theorem A.T2 is welfare maximizing, in particular the discounted expected 
utility of all consumers is equal to maximum attainable welfare, $V(a)=W(a)$, for all cars with positive shares in the stationary fleet age distribution.

Proof. The statements of both corollaries are proven as part of the proof of Theorem A.T2.

Theorem A.T2 shows how closely the optimal car replacement problem (48) is connected to the optimal holding/trading problem (41). The optimal replacement problem provides both the equilibrium scrappage threshold $\gamma^{\star}$ and the price function $P(a)$ defined as shadow prices of what happens to be equivalent to the welfare maximizing social planner's problem.

With the adjustment of the physical transition matrix $Q$ introduced in Theorem A.T2, the stationary holdings distribution $q\left(\gamma^{\star}\right)$ takes the form

$$
q\left(\gamma^{\star}\right)=\left(q(1), \ldots, q\left(\gamma^{\star}\right), 0, \ldots, 0, q(A)\right)
$$

where no cars of age $a \in\left\{\gamma^{\star}+1, \ldots, A-1\right\}$ exist the economy. The stationary age distribution $q\left(\gamma^{\star}\right)$ can be computed by successive approximations by taking transition matrix $Q\left(\gamma^{\star}\right)$ to a large power, or by solving linear system as explained in Appendix C. When the model does not allow for accidents, $\alpha(a)=0, \forall a, Q\left(\gamma^{\star}\right)$ is periodic, leading to the failure of successive approximations method in this case.

Theorem A.T2 provides a way to compute the equilibrium of the homogeneous consumer economy numerically. The straightforward approach amounts to first solving the optimal replacement problem (48) using successive approximations or policy (Newton-Kantorovich) iterations (Rust, 1987). The dimension (number of points in the state space) of this problem is $A$. Afterwards, all necessary components of the equilibrium can be computed from $W(a)$ following Theorem A.T2. There is, however, a much more efficient numerical algorithm for finding the equilibrium of the homogeneous consumer economy which does not require fixed point solution, and instead amounts to solving a series of linear equation systems of a lower than $A$ dimension.

We conclude the section with the presentation of the fast algorithm for finding stationary equilibrium in the homogeneous consumer economy defined in Theorem A.T2. The main idea of the algorithm is to express the indifference condition from Corollary A.C1 as the system of $\gamma^{\star}-1$ linear equations to determine the unrestricted prices $P(a), a \in\left\{1, \ldots, \gamma^{\star}-1\right\}$.

Because by Corollary A.C1 consumers are effectively indifferent between any dynamic trading strategies, the strategy of perpetual replacing an existing car of age $a$ results in the maximum attainable expected discounted utility $V(a)$. We have for $a \in\left\{1, \ldots, \gamma^{\star}-1\right\}$

$$
V(a)=\frac{1}{1-\beta}(u(a)-\beta \mu[P(a)-(1-\alpha(a)) P(a+1)-\alpha(a) \underline{P}]) .
$$

Let $V(0)$ denote the value of having a new car which is measured right after trading instead of the beginning of the period. Then (55) also holds for $a=0$, and $V(0)=W(0)$. Thus, using $V(a)-V(a+1)=W(a)-W(a+1)$ and the definition of the price function (50), we have for $a \in\left\{0, \ldots, \gamma^{\star}-2\right\}$

$$
V(a)-\mu P(a)=V(a+1)-\mu P(a+1),
$$

which leads to the following linear equation in prices $(P(a), P(a+1), P(a+2))$ :

$$
\begin{aligned}
\mu P(a)+\mu(\alpha(a) \beta-\beta-1) P(a+1)+\mu \beta(1 & -\alpha(a+1)) P(a+2)= \\
& =u(a)-u(a+1)+\beta \mu \underline{P}(\alpha(a)-\alpha(a+1)) .
\end{aligned}
$$


The collection of equations (57) for $a \in\left\{0, \ldots, \gamma^{\star}-2\right\}$ forms the system of $\gamma^{\star}-1$ equations with $\gamma^{\star}-1$ unknowns, and can be easily solved numerically under our assumption that $u(a+1) \leq$ $u(a)$ for $a \in 0, \ldots, A$. The system be written in matrix form as

$$
X \cdot P=Y,
$$

where $P$ is the column vector of prices $\{P(a)\}_{a \in\left\{1, \ldots, \gamma^{\star}-1\right\}}$,

$$
\begin{aligned}
& X=\left[\begin{array}{ccccccc}
\alpha(0) \beta-\beta-1 & \beta-\alpha(1) \beta & 0 & 0 & \cdots & 0 & 0 \\
1 & \alpha(1) \beta-\beta-1 & \beta-\alpha(2) \beta & 0 & \cdots & 0 & 0 \\
0 & 1 & \alpha(2) \beta-\beta-1 & \beta-\alpha(3) \beta & \cdots & 0 & 0 \\
0 & 0 & 1 & \alpha(3) \beta-\beta-1 & \cdots & 0 & 0 \\
0 & 0 & 0 & 1 & \cdots & 0 & \vdots \\
\vdots & \vdots & \vdots & \vdots & \ddots & \vdots & 0 \\
0 & 0 & 0 & 0 & \cdots & \alpha\left(\gamma^{\star}-3\right) \beta-\beta-1 & \beta-\alpha\left(\gamma^{\star}-2\right) \beta \\
0 & 0 & 0 & 0 & \cdots & 1 & \alpha\left(\gamma^{\star}-2\right) \beta-\beta-1
\end{array}\right] \\
& Y=\left[\begin{array}{c}
(u(0)-u(1)) / \mu+\beta \underline{P}(\alpha(0)-\alpha(1))-\bar{P} \\
(u(1)-u(2)) / \mu+\beta \underline{P}(\alpha(1)-\alpha(2)) \\
u(2)-u(3)) / \mu+\beta \underline{P}(\alpha(2)-\alpha(3)) \\
\vdots \\
\left(u\left(\gamma^{\star}-3\right)-u\left(\gamma^{\star}-2\right)\right) / \mu+\beta \underline{P}\left(\alpha\left(\gamma^{\star}-3\right)-\alpha\left(\gamma^{\star}-2\right)\right) \\
\left(u\left(\gamma^{\star}-2\right)-u\left(\gamma^{\star}-1\right)\right) / \mu+\beta \underline{P}\left(\alpha\left(\gamma^{\star}-2\right)-1\right)
\end{array}\right] .
\end{aligned}
$$

Note that the dimension of the system (58) is dependent on the equilibrium scrappage age $\gamma^{\star}$. Consequently, it has to be known beforehand to solve for the equilibrium prices. Yet, by Theorem A.T2 it is only determined as part of the solution of the replacement problem (48), which the fast algorithm is aiming to avoid. The alternative approach requires an iterative calculation, where the system is solved for various values of $\gamma^{\star}$ until the equilibrium is found. Because solving a system of linear equations implies much lower computational cost than finding a fixed point of Bellman operator (41), and in particular because the dimensionality of the latter problem $(A)$ is larger than that of the linear system, the proposed algorithm is more computationally efficient. In the next Section, as it will be clear, there is no corresponding optimal replacement problem, and our computational approach relies on the same algorithm.

Note that system (58) is well defined for any $\gamma^{\star} \in\{2, \ldots, A\}$, and consequently an equilibrium price vector $P\left(a, \gamma^{\star}\right)$ can be computed for any value of the optimal scrappage age. Therefore it is necessary to establish the formal condition for the iterative algorithm to identify the equilibrium solution.

Lemma A.L1 (Conditions for equilibrium computation). Let $P(a, \zeta)$ be the solution to the system of linear equations (57) for $a \in\{0, \ldots, \zeta-2\}$, for some $\zeta \in\{2, \ldots, A\}$. The necessary and sufficient condition for $P(a)$ and $\zeta$ to constitute equilibrium price function and scrappage age defined in Theorem A.T2, is that the following two statements hold simultaneously:

$$
\begin{aligned}
\forall a \in\{1, \ldots, \zeta-1\}: & \quad \underline{P} \leq P(a, \zeta) \leq \bar{P} \\
\exists a \in\{1, \ldots, \zeta\}: & P(a, \zeta+1)<\underline{P} .
\end{aligned}
$$

Proof. Consider the policy-specific value function $w(a, \zeta)$ of the optimal replacement problem (48). It can be derived by induction starting from the case $a=\zeta$ where $w(\zeta, \zeta)=W(0)-\mu[\bar{P}-$ $\underline{P}]=W\left(\gamma^{\star}\right)$. Here $\gamma^{\star}=\delta^{\star}$ defined in (49) is the optimal replacement age that coincides with equilibrium scrappage age by Theorem A.T2. By definition of $\delta^{\star}$ it also holds that $W\left(\gamma^{\star}\right)=$ $W(a)$ for $a \in\left\{\gamma^{\star}, \ldots, A\right\}$ where replacement choice is optimal. For $a \in\{0, \ldots, \zeta-1\}$ function 
$w(a, \zeta)$ is given by:

$$
\begin{aligned}
w(a, \zeta)=\sum_{k=0}^{\zeta-a-1} \beta^{k}(1-\alpha(a+k-1))^{k}[u(a+k)+\alpha(a+k) \beta W(A)]+ & \\
& +\beta^{\zeta-a}(1-\alpha(a-1))^{\zeta-a} W\left(\gamma^{\star}\right) .
\end{aligned}
$$

The way that the system (58) is constructed establishes a one-to-one correspondence between its solution $P(a, \zeta)$ for given $\zeta$ and the policy-specific value functions $w(a, \zeta)$ given by:

$$
P(a, \zeta)=\bar{P}-\frac{1}{\mu}[W(0)-w(a, \zeta)]
$$

where we excluded the case of immediate replacing of a new car, so $\zeta>0$ and $w(0, \zeta)=$ $W(0)$. It is easy to verify that prices given by (62) for a fixed $\zeta$ constitute a solution to the corresponding system of equations, and conversely the solution of the system gives rise to $w(a, \zeta)$ that is consistent with the solution of the optimal replacement model (48), such that (61) is satisfied.

We are now in position to prove the statement of the lemma. Necessity follows from, first, observing that when $\zeta=\gamma^{\star}, w(a, \zeta)=W(a)$ and condition (59) is satisfied due to monotonicity of $W(a)$. Next, computing (61) at the point $a=\gamma^{\star}, \zeta=\gamma^{\star}+1$ we obtain:

$$
w\left(\gamma^{\star}, \gamma^{\star}+1\right)=u\left(\gamma^{\star}\right)+\beta\left(1-\alpha\left(\gamma^{\star}\right)\right) W\left(\gamma^{\star}+1\right)+\alpha\left(\gamma^{\star}\right) \beta W(A)<W(0)-\mu[\bar{P}-\underline{P}],
$$

where the last inequality follows directly from the definition of $\gamma^{\star}$ as the optimal replacement threshold. Combing this inequality with (62) leads to (60) which is satisfied for $a=\gamma^{\star}$.

To prove sufficiency first note that conditions (59) and (60) can be reformulated in terms of policy-specific values computed by inverse of (62) from the solution of the linear system, as:

$$
\begin{array}{rc}
\forall a \in\{1, \ldots, \zeta-1\}: & W\left(\gamma^{\star}\right) \leq w(a, \zeta) \leq W(0), \\
\exists a \in\{1, \ldots, \zeta\}: & w(a, \zeta+1)<W\left(\gamma^{\star}\right) .
\end{array}
$$

We need to prove that if (63) and (64) hold, $\zeta$ constitutes the optimal replacement age. In this case then $w(a, \zeta)=W(a)$ for all $a$ and the computed prices indeed coincide with equilibrium prices due to the similarity between (62) and (50). Again, evaluating $w(\zeta, \zeta+1)$ we establish from (64) that replacement rather than keeping is the optimal choice in the problem (48) at age $\zeta$. From the first inequality in (63) if follows that the opposite is true for $\zeta-1$. Thus, $\zeta$ satisfies definition of the optimal replacement age (49).

Lemma A.L1 states that the market equilibrium is determined by the solution $P\left(a, \gamma^{\star}\right)$ with maximum scrappage age $\gamma^{\star}$ such that all prices are located between the scrap value and the price of the new car. An increase of the scrappage age by one year to $\gamma^{\star}+1$ would result in at least one price dipping below the scrap value. The intuition for this results is straightforward: if the cars are forced to stay in the economy longer than their optimal renewal age, an additional monetary compensation ("negative" price below scrap value) must be provided for the owners of those cars that are excessively old.

Lemma A.L1 provides a clear stopping rule for the iterative equilibrium computation algorithm: different values of $\gamma^{\star}$ should be tried until the pair $\left(\gamma^{\star}, \gamma^{\star}+1\right)$ that satisfies conditions (59) and (60) is found. An efficient binary search algorithm would require the number of iterations on the order of $\log _{2} A$ to find the equilibrium. Therefore, it is beneficial to adjust $A$ 
according to the model parameters. For example, solving the static equivalent of the optimal replacement problem (with $\beta=0$ ) necessarily results in higher optimal replacement threshold than the dynamic version, and thus provides a reasonable upper bound.

\section{B Proofs}

\section{B.1 Proof of Theorem 1 (page 14)}

Proof. We start by constructing the ownership distribution $p(P, \bar{a})$ such that (15) is equivalent to the conditions (b)-(d) in equilibrium definition D1 for any $\{\bar{a}, P(a)\}$.

Let $q(\bar{a}, P)$ be invariant distribution of $Q$ given by (12), such that condition (b) in D1 is satisfied. Then the corresponding $p(\bar{a}, P)$ given by (6) is an invariant distribution of $\Omega$ defined in (14) for any $\{\bar{a}, P(a)\}$ which follows from its non-irreducibility. Therefore, it holds that $p=p \Omega$ and the second equality in (15) is satisfied. Further, it follows from (10) that conditions (c) and (d) in D1 can be expressed as a fixed point of matrix $\Delta(P)$, corresponding to the first equality in (15). The final equality in (15) is simple substitution of the first one into the second.

It is now left to show the existence of scrappage age $\bar{a}$ and a price vector $P(a), a \in$ $\{1, \ldots, \bar{a}-1\}$ such that both (a) in D1 and market clearing conditions are satisfied given the above $p(\bar{a}, P)$. Then all equalities in (15) hold, and due to equivalence shown above, D1 is completely fulfilled.

For a fixed $\bar{a}$, define a mapping $\Lambda(P): R^{\bar{a}-1} \rightarrow R^{\bar{a}-1}$ by $\Lambda(P)=P+E D(P)$, where $R_{+}^{n}$ denotes the positive orthant of $R^{n}$ and $E D(P)$ is defined in (10). From Lemma L1 it follows that $E D$ and thus $\Lambda$ is a continuous mapping from $R^{\bar{a}-1} \rightarrow R^{\bar{a}-1}$. Note also that for any $P$ the components of $E D(P)$ lie in the interval $[-1,1]$. Thus, when prices are sufficiently high, a vanishing number of consumers will wish to buy any new car but nearly all consumers will want to sell their cars, so $E D(P)$ will be close to a vector with all its components equal to -1 . Similarly, for a sufficiently low set of prices (possibly negative), nearly all consumers will wish to buy used cars and very few will want to sell their vehicles at such low prices. So for such prices $E D(P)$ will be close to a vector with all of its components equal to +1 . It follows that we can define a compact box $B$ in $R^{\bar{a}-1}$ where $\Lambda$ satisfies an "inward pointing" property on the boundaries of this box, so it follows that $\Lambda: B \rightarrow B$. Since $\Lambda$ is a continuous mapping and $B$ is a compact, convex set, the Brouwer fixed point theorem implies that a fixed point of $\Lambda$ exists, and it is clear that any such fixed point satisfies $E D(P)=0$. Using this set of prices, the fraction of consumers without cars is given by (11), and the condition $D(0, P)=S(\bar{a}, P)$ in D1 is satisfied due to the normalization of the choice probabilities in supply of scrap cars and demand of new cars. Finally, it is possible to show that for small enough $\bar{a}$ conditions (a) of D1 are always satisfied, whereas when $\bar{a}$ is too big, the corresponding price function may violate them.

\section{B.2 Proof of Lemma L1 (page 16)}

Proof. This follows from the implicit function theorem provided we can show that 1) $\Gamma$ is a continuously differentiable function of $P$, and 2) the $(\bar{a}+1) \times(\bar{a}+1)$ matrix $I-\nabla_{E V} \Gamma(E V, P)$ is nonsingular for any $P$ where $E V=\Gamma(E V, P)$, where $\nabla_{E V}$ denotes the gradient operator with respect to $E V$. However by Lemma L2 we have $\nabla_{E V} \Gamma(E V, P)=\beta \Delta(P) \Omega$. This implies that $I-\nabla_{E V} \Gamma(E V, P)$ is nonsingular with inverse equal to

$$
[I-\beta \Delta(P) \Omega]^{-1}=\sum_{t=0}^{\infty} \beta^{t}[\Delta(P) \Omega]^{t}
$$


where the latter series is convergent due to the assumption that $\beta \in(0,1)$ and $\|\Delta(P) \Omega\|=1$. It is easy to show that $\nabla_{P} \Gamma(E V, P)$ exists and is continuous in $E V$ and $P$ using the formulas for $\Gamma$ in equation (3). Thus, by the implicit function theorem $E V(P)$ is a continuously differentiable function of $P$ with gradient given by $\nabla_{P} E V(P)=[I-\beta \Delta(P) \Omega]^{-1} \nabla_{P} \Gamma(E V, P)$.

\section{B.3 Proof of Lemma L2 (page 17)}

Proof. This can be shown via algebra after differentiating the $\Gamma$ operator with respect to $E V$ in equations (1)-(3) and expressing the result as a matrix product.

\section{B.4 Proof of Theorem 2 (page 21)}

Proof. The proof is analogous to B.1. We show that the conditions (22) and (23) are equivalent to the the conditions (b)-(d) in equilibrium definition D1 for any $\{\bar{a}, P(a)\}$, and then apply the Brouwer fixed point theorem in the same way (the latter step is skipped here for space considerations).

First, allow $q_{\tau}(\varnothing)$ be chosen such that it holds $D(\varnothing, P)-S(\varnothing, P)$. This can be achieved by applying formula (11) for each particular consumer type $\tau$. Then (23) has exactly one invariant distribution, which structure is given by (18) and which therefore defines $q_{\tau}$.

Next, to show that the collection of $q_{\tau}$ form the overall holdings distribution that satisfy (22), note that with the structure of ownership distribution $p$ given in (18)-(25) equation (21) can be written as:

$$
\sum_{\tau=1}^{N_{\tau}} f(\tau) p_{\tau}(P, \bar{a})\left(\Delta_{\tau}(P)-I\right)=(E D(P), D(0, P)-S(\bar{a}, P), D(\varnothing, P)-S(\varnothing, P)) .
$$

In equilibrium $E D(P)=(0, \ldots, 0), D(\varnothing, P)=S(\varnothing, P)$ due to how $q_{\tau}(\varnothing)$ was chosen above, and the last component of the righ hand side of $(66)$ is zero because the choice probabilities add up to one. It follows:

$$
\sum_{\tau=1}^{N_{\tau}} f(\tau) p_{\tau}(P, \bar{a}) \Delta_{\tau}(P)=\sum_{\tau=1}^{N_{\tau}} f(\tau) p_{\tau}(P, \bar{a}) .
$$

Multiplying both side of (67) with matrix $\Omega$ on the right, and using the fact that $p_{\tau}(P, \bar{a})$ is a fixed point of $\Delta_{\tau}(P) \Omega$ in the left hand side, gives:

$$
\sum_{\tau=1}^{N_{\tau}} f(\tau) p_{\tau}(P, \bar{a})=\left[\sum_{\tau=1}^{N_{\tau}} f(\tau) p_{\tau}(P, \bar{a})\right] \Omega
$$

Thus, $\sum_{\tau=1}^{N_{\tau}} f(\tau) p_{\tau}(P, \bar{a})$ is an invariant distribution of $\Omega$, which implies that $q(P, \bar{a})$ in (22) is an invariant distribution of $Q$ due to (14).

\section{B.5 Proof of Lemma L3 (page 22)}

Proof. The uniqueness of the invariant distribution $q_{\tau}(P)=q_{\tau}(P) \Delta_{\tau}(P) \Omega$ established in Section B.4 enables us to write $q_{\tau}(P)$ as the solution to a system of linear equations that is related to, but distinct from the linear system (24) with the key difference being that the matrix in this related system of linear equations is invertible for each $P$. This means that we can write $q_{\tau}(P)$ explicitly in terms of the inverse of this matrix, which can be shown by inspection to be a function of $P$ since it depends on $P$ only via the matrix $\Delta_{\tau}(P)$ which is continuously differentiable 
in $P$ by Lemma L1. Hence, using matrix calculus we can write an explicit formula for the gradient $\nabla_{P} q_{\tau}(P)$ which we use to implement Newton's method to solve the system of equations $E D(P)=0$. The details of this are contained in Appendix C.

\section{B.6 Proof of Theorem 3 (page 23)}

Proof. The proof is analogous to B.1 and B.4. We show that the conditions (26) and (27) are equivalent to the the conditions (b)-(d) in equilibrium definition D1 for any $\{\bar{a}, P(a)\}$, and then apply the Brouwer fixed point theorem in the same way (again, the latter step is skipped here for space considerations).

Similar to B.4, first allow $q_{y}(\varnothing)$ be chosen such that it holds $D(\varnothing, P)-S(\varnothing, P)$. This can be achieved by applying formula (11) for each income level $y$. Then, together with holdings distribution $q(\bar{a}, P)$ which we take to be invariant distribution of $Q$ given by (12), the equilibrium ownership distribution is completely characterized by (25). With this ownership distribution condition (b) in D1 is satisfied.

Next, repeating the argument from B.4, we can characterize the market clearing conditions using the equation:

$$
\sum_{y} \pi(y) p_{y}(P, \bar{a})\left(\Delta_{y}(P)-I\right)=(E D(P), D(0, P)-S(\bar{a}, P), D(\varnothing, P)-S(\varnothing, P)) .
$$

In order to show that the right hand side only contains zeros with the chosen ownership distribution, multiply the left hand side by $\Omega \Omega^{-1}$ to get the expression:

$$
\begin{aligned}
& {\left[\sum_{y} \pi(y) p_{y}(P, \bar{a}) \Delta_{y}(P) \Omega-\sum_{y} \pi(y) p_{y}(P, \bar{a}) \Omega\right] \Omega^{-1}=} \\
& \quad\left[(q(P, \bar{a})[1-q(\varnothing)], q(\varnothing)) \sum_{y} \pi(y)-(q(P, \bar{a})[1-q(\varnothing)], q(\varnothing)) \Omega\right] \Omega^{-1}= \\
& \quad(0, \ldots, 0) \Omega^{-1}=(0, \ldots, 0) .
\end{aligned}
$$

The expression is simplified using (26) and (27) and the fact that the (income specific) chosen ownership probabilities constitute the fixed points of the physical transition matrix $\Omega$.

\section{B.7 Proof of Theorem 4 (page 32)}

Proof. The holdings and ownership distributions given by (31) and (32), physical transition matrix is given by (33), and the trade transition matrix by (34) are appropriate generalizations of the same objects in the three cases the theorem is addressing. It is straightforward to check that the proofs of all three theorems follow through with these redefined distributions and transition matrices, taking into account the argument about the only significant change having to do with additional $J-1$ unknowns (market shares) determined in $J-1$ additional equations given in (36).

\section{Gradient Of Invariant Distribution}

In this appendix we consider the general problem of calculating the derivative of an invariant distribution with respect to parameters affecting a Markov transition matrix. Let the parameters 
be $\theta$ (in our application $\theta$ is a vector of prices of cars in a secondary market equilibrium) and consider a Markov transition probability matrix $P(\theta)$ that depends on these parameters in a continuously differentiable fashion. Thus, we assume that the mapping $\nabla_{\theta} P(\theta)$ from $R^{k}$ to $R^{k * n * n}$ (where the latter can be interpreted as the space of $k$-tuples of $n \times n$ matrices) exists and is a continuous function of $\theta$. To make things easier to understand, assume initially that $k=1$ so we are considering $P(\theta)$ and $q(\theta)$ as functions of a single parameter $\theta$. If $\theta$ has $k$ components (i.e. $\theta \in R^{k}$ ) we simply "stack" the formulas we provide below in the univariate case into a $k$-tuple.

We are interested in determining the conditions under which $q(\theta)$, the unique invariant distribution of $P(\theta)$, is a continuously differentiable function of $\theta$ and, if so, to find an expression for $\nabla_{\theta} q(\theta)$. The invariant distribution $q(\theta)$ satisfies the equation

$$
q(\theta)=q(\theta) P(\theta),
$$

which can be recast as $q(\theta)$ being a left zero of the matrix $I-P(\theta), q(\theta)[I-P(\theta)]=0$. The usual application of the Implicit Function Theorem applies when $q(\theta)$ can be written as a zero of some continuously differentiable nonlinear mapping $F(q, \theta)=0$ with the added condition that $\nabla_{q} F(q, \theta)$ is nonsingular at a zero of $F$. Then the Implicit Function Theorem guarantees that there is a continuously differentiable function $q(\theta)$ in a neighborhod of this zero, and we have

$$
\nabla_{\theta} q(\theta)=-\left[\nabla_{q} F(q(\theta), \theta)\right]^{-1} \nabla_{\theta} F(q(\theta), \theta) .
$$

However this usual application of the Implicit Function Theorem is inapplicable because in this case $\nabla_{q} F(q, \theta)=I-P(\theta)$ and this matrix is singular (note that if $e$ is a vector of ones, then $[I-P(\theta)] e=0$ where 0 is a vector of zeros). Thus, we have to approach this problem from a different angle.

When the invariant distribution is unique, it can be shown that $q(\theta)^{\prime}$, the $n \times 1$ transpose of $q(\theta)$, is the unique solution to the expanded $(n+1) \times(n+1)$ linear system given by

$$
\left[\begin{array}{cc}
I-P(\theta)^{\prime} & e \\
e^{\prime} & 1
\end{array}\right]\left[\begin{array}{c}
q(\theta)^{\prime} \\
1
\end{array}\right]=\left[\begin{array}{l}
e \\
2
\end{array}\right]
$$

where $e$ is an $n \times 1$ vector all of whose elements equal 1. Thus, the matrix on the right hand side of equation (72) is invertible and we can write

$$
\left[\begin{array}{c}
q(\theta)^{\prime} \\
1
\end{array}\right]=\left[\begin{array}{cc}
I-P(\theta)^{\prime} & e \\
e^{\prime} & 1
\end{array}\right]^{-1}\left[\begin{array}{l}
e \\
2
\end{array}\right] .
$$

Let $A(\theta)$ be the $(n+1) \times(n+1)$ matrix on the right hand side of equation (72). Then we have that $\nabla_{\theta} q(\theta)^{\prime}$ is the upper left $n \times n$ submatrix of the product of $\nabla_{\theta} A^{-1}(\theta)$ times the vector $\left(e^{\prime} 2\right)^{\prime}$. Further, we use the following formula for the gradient of $A^{-1}(\theta)$ with respect to $\theta$

$$
\nabla_{\theta} A^{-1}(\theta)=-A^{-1}(\theta)\left[\nabla_{\theta} A(\theta)\right] A^{-1}(\theta) .
$$




\section{NMNL Choice Probabilities And Expected Value Functions}

We assume that the multivariate distrubtion of $\varepsilon$ is generalized extreme value family and thus has joint $\mathrm{CDF}$ of the form

$$
F\left(\varepsilon_{1}, \ldots, \varepsilon_{n}\right)=\exp \left\{-G\left(e^{-\varepsilon_{1}}, \ldots, e^{-\varepsilon_{n}}\right)\right\}
$$

where the function $G$ maps the positive orthant of $R^{n}$ into the positive real line, and satisfies several properties, including homogeneity of degree $\mu$ for some $\mu>0$. Note that the CDF of a collection of IID Type 1 extreme value random variables is a special case of equation (75) when $G\left(y_{1}, \ldots, y_{n}\right)=\sum_{i=1}^{n} y_{i}$.

In the auto problem, we index the arguments of the joint CDF as $\left(\varepsilon_{\kappa}, \varepsilon_{0},\left\{\varepsilon_{d, j}\right\}\right)$. Thus, $F\left(\varepsilon_{\emptyset}, \varepsilon_{0},\left\{\varepsilon_{d, j}\right\}\right)$ is the CDF of these preference shocks and we assume it has the representation

$$
F\left(\varepsilon_{\varnothing}, \varepsilon_{\kappa},\left\{\varepsilon_{d, j}\right\}\right)=\exp \left\{-G\left(e^{-\varepsilon_{\varnothing}}, e^{-\varepsilon_{\kappa}},\left\{e^{-\varepsilon_{d, j}}\right\}\right)\right\}
$$

where the function $G\left(y_{\varnothing}, y_{0},\left\{y_{d, j}\right\}\right)$ is given by

$$
G\left(y_{\varnothing}, y_{0},\left\{y_{d, j}\right\}\right)=y_{\varnothing}^{\frac{1}{\sigma}}+y_{0}^{\frac{1}{\sigma}}+\left(\sum_{j=1}^{J}\left(\sum_{d=0}^{\bar{a}_{j}-1} y_{d, j} \frac{1}{\sigma_{j}}\right)^{\frac{\sigma_{j}}{\sigma_{j>0}}}\right)^{\frac{\sigma_{j>0}}{\sigma}} .
$$

where $\sigma, \sigma_{j>0}$ and $\left\{\sigma_{j}\right\}, j=1, \ldots, J$ are $J+2$ "scale/similarity parameters" of the GEV distribution that implies the nesting structure outlined i Figure 4.

Note that the parameter $\sigma$ controls the level of variability of the preference shocks in the top level of the choice tree. As $\sigma \rightarrow 0$ the overall scale of the preference shocks tend to zero due to the inequalities

$$
\sigma \geq \sigma_{j>0} \geq \sigma_{j} \geq 0 \quad j=1, \ldots, J
$$

that must be satisfied for $F$ to be a valid CDF. Hence, the similarity parameter $\sigma_{j>0}$ scaling the shocks at the upper level of this subtree, as well as the car type specific similarity parameters $\sigma_{j}$ discussed above. If we impose the restriction that $\sigma=\sigma_{j>0}$, then the three level NMNL choice tree collapses to the two level choice tree structure similar to Figure 4 where the scale parameters preference shocks for the top level choices are the same, and we only allow different scale parameters $\sigma_{j}$ to reflect correlation/similarity in different ages of the them type $j$ of car. If we further restrict that $\sigma=\sigma_{j>0}=\sigma_{j}$ for $j=1, \ldots, J$, then the NMNL collapses to a standard MNL model, where there is a common scale parameter $\sigma$ for all preference shocks, but these shocks are IID.

When $\varepsilon$ is a vector of IID GEV preference shocks, taking the expectation of both sides of (28), and using max-stability property of the generalized extreme value distribution (McFadden, 1981 ), leads to the well known nested "log-sum" expression for $E V(a) \equiv \int V(a, \varepsilon) d F(\varepsilon), a \in$ $\{1, \ldots, \bar{a}-1\}$ :

$$
E V_{\tau}(\varnothing)=\sigma \log \left(\exp \left\{v_{\tau}(\varnothing, \varnothing) / \sigma\right\}+\exp \left\{I_{j>0}(\varnothing) / \sigma\right\}\right)
$$

where $I_{j>0}(\varnothing)$ is the inclusive value or ex ante expected maximized utility corresponding to the choice of moving out of the no car state and buying some car $(d, j)$ that provides the consumer the ex post highest discounted utility after observing the preference shocks for each 
$(d, j)$ alternative, and is given by

$$
I_{j>0}(\varnothing)=\sigma_{j>0} \log \left(\sum_{j=1}^{J} \exp \left\{I_{j}(\varnothing) / \sigma_{J>0}\right\}\right) .
$$

where $I_{j}(\varnothing)$ is the inclusive value or expected maximal discounted utility associated with the choice of a particular car type $j$ by an individual who does not own a car, and is given by

$$
I_{j}(\varnothing)=\sigma_{j} \log \left(\sum_{d=0}^{\bar{a}_{j}-1} \exp \left\{v_{\tau}(d, j, \varnothing) / \sigma_{j}\right\}\right) .
$$

The expected value of state $(a, j)$ is $E V_{\tau}(a, j)$ given by

$E V_{\tau}(a, j)= \begin{cases}\sigma \log \left(\exp \left\{v_{\tau}(\varnothing, a, j) / \sigma\right\}+\exp \left\{I_{j>0}(a, j) / \sigma\right\}\right) & \text { if } a=\bar{a}_{j} \\ \sigma \log \left(\exp \left\{v_{\tau}(\varnothing, a, j) / \sigma\right\}+\exp \left\{v_{\tau}(\kappa, a, j) / \sigma\right\}+\exp \left\{I_{j>0}(a, j) / \sigma\right\}\right) & \text { otherwise }\end{cases}$

where the first equation for $E V_{\tau}(a, j)$ in (82) is for the case where the current car is at the scrappage age threshold, $a=\bar{a}_{j}$, so keeping this car is no longer an option, and the second formula is for the case where $a<\bar{a}_{j}$ so the consumer has the additional option to choose to keep the current car $(a, j)$ rather than trade it, which has the value $v_{\tau}(\kappa, a, j)$. The inclusive value for the decision to trade for another car is $I_{j>0}(a, j)$ given by

$$
I_{j>0}(a, j)=\sigma_{j>0} \log \left(\sum_{j^{\prime}=1}^{J} \exp \left\{I_{j^{\prime}}(a, j) / \sigma_{j>0}\right\}\right)
$$

where $I_{j^{\prime}}(a, j)$ is the inclusive value associated with the decision to trade the current car $(a, j)$ for some car $\left(d^{\prime}, j^{\prime}\right)$ of car type $j^{\prime}$ given by

$$
I_{j^{\prime}}(a, j)=\sigma_{j^{\prime}} \log \left(\sum_{d^{\prime}=0}^{\bar{a}_{j^{\prime}}-1} \exp \left\{v_{\tau}\left(d^{\prime}, j^{\prime}, a, j\right) / \sigma_{j^{\prime}}\right\}\right) .
$$

For notational simplicity, we suppressed the dependence of the value functions on the set of car prices. However, to describe equilibrium it is now necessary to make the dependence on prices $P$ explicit when deriving the nested logit choice probabilities below. First consider the probability that the person chooses to remain in the no car state

$$
\Pi_{\tau}(\varnothing \mid \varnothing, P)=\frac{\exp \left\{v_{\tau}(\varnothing, \varnothing) / \sigma\right\}}{\exp \left\{v_{\tau}(\varnothing, \varnothing) / \sigma\right\}+\exp \left\{I_{j>0}(\varnothing) / \sigma\right\}}
$$

Let $\Pi_{\tau}(d, j \mid \varnothing, P)$ be the probability that the consumer chooses to buy a car $(d, j)$. This is given by the product of "transition probabilities" reflecting the three level choice tree

$$
\Pi_{\tau}(d, j \mid \varnothing, P)=\Pi_{\tau}(d \mid j, \varnothing, P) \Pi_{\tau}(j \mid j>0, \varnothing, P) \Pi_{\tau}(j>0 \mid \varnothing, P)
$$

where $\Pi_{\tau}(j>0 \mid \varnothing, P)$ denotes the probability that the consumer chooses some type of car,

$$
\Pi_{\tau}(j>0 \mid \varnothing, P)=1-\Pi_{\tau}(\varnothing \mid \varnothing, P)=\frac{\exp \left\{I_{j>0}(\varnothing) / \sigma\right\}}{\exp \left\{v_{\tau}(\varnothing, \varnothing) / \sigma\right\}+\exp \left\{I_{j>0}(\varnothing) / \sigma\right\}}
$$


and $\Pi_{\tau}(j \mid j>0, \varnothing, P)$ is the probability the consumer chooses car type $j$ given the decision to choose some type of car,

$$
\Pi_{\tau}(j \mid j>0, \varnothing, P)=\frac{\exp \left\{I_{j}(\varnothing) / \sigma_{j>0}\right\}}{\sum_{j^{\prime}=1}^{J} \exp \left\{I_{j^{\prime}}(\varnothing) / \sigma_{j>0}\right\}}
$$

and $\Pi_{\tau}(d \mid j, \varnothing, P)$ is the probability the consumer chooses a car of age $d$ given that they chose a car of type $j$

$$
\Pi_{\tau}(d \mid j, \varnothing, P)=\frac{\exp \left\{v_{\tau}(d, j, \varnothing, P) / \sigma_{j}\right\}}{\sum_{d^{\prime}=0}^{\bar{a}_{j}-1} \exp \left\{v_{\tau}\left(d^{\prime}, j, \varnothing, P\right) / \sigma_{j}\right\}} .
$$

Now consider a consumer who has a car $(a, j)$ of age $a$ and type $j$. The probability of the choice $d=\varnothing$ (i.e. to sell their current car and not replace it with another one) is given by

$$
\Pi_{\tau}(\varnothing \mid a, j, P)= \begin{cases}\frac{\exp \left\{v_{\tau}(\varnothing, a, j, P) / \sigma\right\}}{\exp \left\{v_{\tau}(\emptyset, a, j, P) / \sigma\right\}+\exp \left\{v_{\tau}(\kappa, a, j, P) / \sigma\right\}+\exp \left\{I_{j>0}(a, j) / \sigma\right\}} & \text { if } a<\bar{a}_{j} \\ \frac{\exp \left\{v_{\tau}(\varnothing, a, j, P) / \sigma\right\}}{\exp \left\{v_{\tau}(\varnothing, a, j, P) / \sigma\right\}+\exp \left\{I_{j>0}(a, j) / \sigma\right\}} & \text { if } a=\bar{a}_{j}\end{cases}
$$

which reflects the constraint that for any car type $j$ a consumer who holds the oldest possible age $a=\bar{a}_{j}$ is forced to scrap it, so we have

$$
\Pi_{\tau}(0 \mid a, j, P)= \begin{cases}\frac{\exp \left\{v_{\tau}(\kappa, a, j, P) / \sigma\right\}}{\exp \left\{v_{\tau}(\varnothing, a, j, P) / \sigma\right\}+\exp \left\{v_{\tau}(\kappa, a, j, P) / \sigma\right\}+\exp \left\{I_{j>0}(a, j) / \sigma\right\}} & \text { if } a<\bar{a}_{j} \\ 0 & \text { if } a=\bar{a}_{j}\end{cases}
$$

Since there are only three possible choices at the top level of the choice tree in Figure 4, it follows that the probability of choosing to trade the current car $(a, j)$ for some other car $\left(d, j^{\prime}\right)$ is given by

$$
\Pi_{\tau}(j>0 \mid a, j, P)=1-\Pi_{\tau}(\varnothing \mid a, j, P)-\Pi_{\tau}(0 \mid a, j, P)
$$

which is valid for any value of $a \in\left\{1, \ldots, \bar{a}_{j}\right\}$. Let $\Pi_{\tau}\left(d, j^{\prime} \mid a, j, P\right)$ be the probability the consumer trades their current car $(a, j)$ for some other car $\left(d, j^{\prime}\right)$. Similar to the probability $\Pi_{\tau}\left(d, j^{\prime} \mid \varnothing, P\right)$ given in equation (86) above, this probability can be written as a product of three "transition probabilities"

$$
\Pi_{\tau}\left(d, j^{\prime} \mid a, j, P\right)=\Pi_{\tau}\left(d \mid j^{\prime}, a, j, P\right) \Pi_{\tau}\left(j^{\prime} \mid j>0, a, j, P\right) \Pi_{\tau}(j>0 \mid a, j, P)
$$

where the probability of choosing to trade the current car $(a, j)$ for some other age and type of car is $\Pi_{\tau}(j>0 \mid a, j, P)$ given in equation (92), and $\Pi_{\tau}\left(j^{\prime} \mid j>0, a, j, P\right)$ is the probability the consumer chooses car type $j^{\prime}$ given the decision to trade,

$$
\Pi_{\tau}\left(j^{\prime} \mid j>0, a, j, P\right)=\frac{\exp \left\{I_{j^{\prime}}(a, j, P) / \sigma_{j>0}\right\}}{\sum_{j^{\prime}=1}^{J} \exp \left\{I_{j^{\prime}}(a, j, P) / \sigma_{j>0}\right\}},
$$

and $\Pi_{\tau}\left(d \mid j^{\prime}, a, j, P\right)$ is the probability that the consumer chooses a car of age $d$ given the choice of type of car $j^{\prime}$

$$
\Pi_{\tau}\left(d \mid j^{\prime}, a, j, P\right)=\frac{\exp \left\{v_{\tau}\left(d, j^{\prime}, a, j, P\right) / \sigma_{j^{\prime}}\right\}}{\sum_{d^{\prime}=0}^{\bar{a}_{j^{\prime}}-1} \exp \left\{v_{\tau}\left(d^{\prime}, j^{\prime}, a, j, P\right) / \sigma_{j^{\prime}}\right\}} .
$$

